\title{
Chemical tracers of episodic accretion in low-mass protostars ${ }^{\star}$
}

\author{
Ruud Visser ${ }^{1,2}$, Edwin A. Bergin ${ }^{2}$, and Jes K. Jørgensen ${ }^{3}$ \\ ${ }^{1}$ European Southern Observatory, Karl-Schwarzschild-Str. 2, 85748 Garching, Germany \\ e-mail: rvisser@eso.org \\ 2 Department of Astronomy, University of Michigan, 1085 S. University Ave., Ann Arbor, MI 48109-1107, USA \\ 3 Centre for Star and Planet Formation, Niels Bohr Institute \& Natural History Museum of Denmark, University of Copenhagen, \\ Øster Voldgade 5-7, 1350 Copenhagen K., Denmark
}

Received 19 November 2014 / Accepted 16 March 2015

\begin{abstract}
Aims. Accretion rates in low-mass protostars can be highly variable in time. Each accretion burst is accompanied by a temporary increase in luminosity, heating up the circumstellar envelope and altering the chemical composition of the gas and dust. This paper aims to study such chemical effects and discusses the feasibility of using molecular spectroscopy as a tracer of episodic accretion rates and timescales.

Methods. We simulate a strong accretion burst in a diverse sample of 25 spherical envelope models by increasing the luminosity to 100 times the observed value. Using a comprehensive gas-grain network, we follow the chemical evolution during the burst and for up to $10^{5} \mathrm{yr}$ after the system returns to quiescence. The resulting abundance profiles are fed into a line radiative transfer code to simulate rotational spectra of $\mathrm{C}^{18} \mathrm{O}, \mathrm{HCO}^{+}, \mathrm{H}^{13} \mathrm{CO}^{+}$, and $\mathrm{N}_{2} \mathrm{H}^{+}$at a series of time steps. We compare these spectra to observations taken from the literature and to previously unpublished data of $\mathrm{HCO}^{+}$and $\mathrm{N}_{2} \mathrm{H}^{+} 6-5$ from the Herschel Space Observatory.

Results. The bursts are strong enough to evaporate $\mathrm{CO}$ throughout the envelope, which in turn enhances the abundance of $\mathrm{HCO}^{+}$ and reduces that of $\mathrm{N}_{2} \mathrm{H}^{+}$. After the burst, it takes $10^{3}-10^{4} \mathrm{yr}$ for $\mathrm{CO}$ to refreeze and for $\mathrm{HCO}^{+}$and $\mathrm{N}_{2} \mathrm{H}^{+}$to return to normal. The $\mathrm{H}_{2} \mathrm{O}$ snowline expands outwards by a factor of $\sim 10$ during the burst; afterwards, it contracts again on a timescale of $10^{2}-10^{3} \mathrm{yr}$. The chemical effects of the burst remain visible in the rotational spectra for as long as $10^{5} \mathrm{yr}$ after the burst has ended, highlighting the importance of considering luminosity variations when analyzing molecular line observations in protostars. The spherical models are currently not accurate enough to derive robust timescales from single-dish observations. As follow-up work, we suggest that the models be calibrated against spatially resolved observations in order to identify the best tracers to be used for statistically significant source samples.
\end{abstract}

Key words. stars: formation - stars: protostars - circumstellar matter - accretion, accretion disks - astrochemistry

\section{Introduction}

Stars gain most of their mass while deeply embedded in the molecular cloud core out of which they first formed. The accretion rate of matter from the collapsing core onto the star is probably highly variable in time. On cloud scales, turbulence can induce order-of-magnitude variations in the accretion rates onto individual cores (Padoan et al. 2014). Within a given core, variations of similar magnitude are possible from mass-loading onto an embedded disk or pseudo-disk (Zhu et al. 2009; Vorobyov \& Basu 2010). The disk becomes unstable at some point, resulting in a short-lived accretion burst onto the star. Each burst causes a temporary increase in luminosity, heating up the circumstellar envelope and altering the chemical composition of the gas and dust. This paper analyzes such chemical effects and explores how to use molecular lines as a probe of variable accretion rates and timescales.

Variable or episodic accretion is the leading explanation for the wide range of protostellar luminosities observed in largescale surveys (Kenyon et al. 1990; Evans et al. 2009; Kryukova et al. 2012; Fischer et al. 2013; Dunham et al. 2014) and for FUor

\footnotetext{
* Herschel is an ESA space observatory with science instruments provided by European-led Principal Investigator consortia and with important participation from NASA.
}

and EXor outbursts in more evolved pre-main-sequence stars (Herbig 1977; Audard et al. 2014). Episodic accretion can also affect processes such as disk fragmentation (Stamatellos et al. 2011, 2012) and lithium depletion (Baraffe \& Chabrier 2010). It is therefore important to understand the magnitude-frequency distribution and determine how often bursts of a certain intensity occur. Based on large-scale variability surveys, the strongest bursts - with accretion rates of at least 100 times the quiescent value - happen every 5-50 kyr per protostar (Scholz et al. 2013). The spacings between periodic shocks in jets and outflows support quiescent intervals anywhere from $10^{4} \mathrm{yr}$ down to $10 \mathrm{yr}$, separating bursts with an unknown range of accretion rates (Devine et al. 1997; Raga et al. 2002; Arce et al. 2013). Highcadence photometric surveys show luminosity variations at the 5-50\% level on timescales of hours to weeks (Billot et al. 2012; Stauffer et al. 2014), though only some of that may be related to accretion variability (Morales-Calderón et al. 2011; Rebull et al. 2014). Despite the incomplete statistics, it appears that stronger accretion bursts occur less frequently than weaker ones. Such a distribution is supported by numerical simulations (Zhu et al. 2009; Vorobyov \& Basu 2010).

Direct observations of luminosity bursts were traditionally limited to the relatively evolved Class II sources, where the lack of an obscuring dusty envelope makes the bursts visible at optical wavelengths. However, bursts have now also been detected in 
at least five embedded Class I protostars (Audard et al. 2014) and even in one deeply embedded Class 0 source (Safron et al. 2015). The detection of luminosity flares during the earliest phases of star formation lends credence to the notion of episodic accretion as a wide-spread phenomenon.

Accretion bursts have the ability to alter the chemical composition of the circumstellar material. For example, the 2008 burst in EX Lup sparked the production of crystalline silicates (Ábrahám et al. 2009) and boosted the column densities of midinfrared $\mathrm{H}_{2} \mathrm{O}$ and $\mathrm{OH}$ lines (Banzatti et al. 2012). In very lowluminosity embedded protostars, whose envelopes are too cold to produce pure $\mathrm{CO}_{2}$ ice, the detection of double-peaked $15-\mu \mathrm{m}$ absorption profiles is attributed to a higher luminosity at some unknown point in the past (Kim et al. 2011, 2012; see also Poteet et al. 2013).

The chemical effects of embedded accretion bursts primarily result from changes in the temperature of the circumstellar gas and dust. When a protostar enters a burst, the increase in luminosity can alter the $\mathrm{D} / \mathrm{H}$ ratio of water and other species (Owen \& Jacquet 2015). The higher temperatures also lead to the evaporation of some of the icy grain mantles (Lee 2007; Visser \& Bergin 2012, hereafter Paper I; Vorobyov et al. 2013). After the burst ends, the luminosity returns to the quiescent value and the envelope cools down almost instantaneously (Johnstone et al. 2013). However, at typical envelope densities, it takes $10^{3}-$ $10^{5} \mathrm{yr}$ for the gas to freeze back onto the cold dust grains. The abundance profiles are out of equilibrium with the observed luminosity for all that time. This could explain the presence of spatially extended $\mathrm{C}^{18} \mathrm{O}$ in eight out of 16 low-mass protostars (Jørgensen et al. 2015).

The best example to date of episodic accretion chemistry in an embedded protostar is the central gap discovered in spatially resolved observations of $\mathrm{H}^{13} \mathrm{CO}^{+} J=4-3$ in IRAS 15398 (Jørgensen et al. 2013). This distribution is consistent with a picture where $\mathrm{HCO}^{+}$and its isotopologs are destroyed by $\mathrm{H}_{2} \mathrm{O}$ in the inner envelope when the temperature exceeds $100 \mathrm{~K}$. However, the current temperature at the edge of the $\mathrm{H}^{13} \mathrm{CO}^{+}$gap is only $\sim 30 \mathrm{~K}$. The mismatch between the dust temperature and the $\mathrm{H}^{13} \mathrm{CO}^{+}$morphology implies that IRAS 15398 was hotter in the past and that the chemistry is still adjusting to the current cold environment. Based on the freeze-out timescale of $\mathrm{H}_{2} \mathrm{O}$, the simplest explanation for that hotter past is an accretion burst that happened 100-1000 yr ago (Jørgensen et al. 2013).

The aim of the current paper is twofold: to explore episodic accretion chemistry in a sample of 25 spherical envelope models, and to address how certain molecular line ratios can be used as a chronometer of when the most recent accretion burst occurred in any given source. Section 2 introduces the sample and summarizes the available observations, including new Herschel spectra of $\mathrm{HCO}^{+}$and $\mathrm{N}_{2} \mathrm{H}^{+} 6-5$. Section 3 presents the physical and chemical models. Section 4 discusses the chemical effects of an accretion burst and Sect. 5 shows how to use observed line ratios to derive episodic accretion timescales. Lastly, Sect. 6 presents the main conclusions.

\section{Source sample and observations}

Paper I explored the chemical aspects of episodic accretion with a set of single-point models at representative densities of $10^{5}$, $10^{6}$, and $10^{7} \mathrm{~cm}^{-3}$. The current paper analyzes the chemistry in a sample of observationally determined spherical protostellar envelope models, each covering a range of densities and temperatures. We use the predicted abundance profiles to simulate molecular line spectra and identify observable diagnostics.
The envelope models are based on the 29 low-mass embedded protostars targeted in the key program "Water in starforming regions with Herschel" (WISH; van Dishoeck et al. 2011). Source coordinates, distances, and other basic properties are listed in Table 1 of Kristensen et al. (2012). In order to identify the best observable diagnostics, we searched the literature for observations of molecular lines and ice column densities. The most widely available tracers are $\mathrm{C}^{18} \mathrm{O} J=2-1,3-2$, and 5-4 (20, 26, and 15 sources); $\mathrm{H}^{13} \mathrm{CO}^{+} 1-0,3-2$, and 4-3 (18, 17, and 16); and $\mathrm{N}_{2} \mathrm{H}^{+} 1-0$ (21). In addition, column densities of $\mathrm{CO}_{2}, \mathrm{CO}$, and $\mathrm{H}_{2} \mathrm{O}$ ice are available for 14,10 , and 12 sources. Lastly, we present previously unpublished spectra of $\mathrm{HCO}^{+} 6-5$ and $\mathrm{N}_{2} \mathrm{H}^{+} 6-5$ (16 and 7 sources) obtained with the Heterodyne Instrument for the Far-Infrared (HIFI; de Graauw et al. 2010) on the Herschel Space Observatory (Pilbratt et al. 2010). The full data set is summarized in Tables 1 and 2. Appendix A offers more details on the observations and the data reduction.

For lack of molecular line observations, we omit NGC 1333 IRAS3, IRAS 12496, R CrA IRS5, and HH100 IRS from the WISH target list. Our final sample therefore contains 25 protostars, including IRAS 15398 (Sect. 1; Jørgensen et al. 2013). These sources have bolometric luminosities from 0.8 to $35.7 L_{\odot}$ and span the full evolutionary sequence from deeply embedded Class 0 to late Class I (Lada 1999).

\section{Model description}

\subsection{Physical framework}

Spherical density and temperature profiles are available from Kristensen et al. (2012) for our entire sample of 25 protostars. The density follows a power law, $n\left(\mathrm{H}_{2}\right) \propto r^{-p}$, where the slope $p$ is one of three free parameters in a grid of models from the one-dimensional continuum radiative transfer program DUSTY (Ivezić et al. 1999; Jørgensen et al. 2002). The other two are the mass and size of the envelope. Kristensen et al. compared the model grid output to the observed spectral energy distributions (SEDs) and submillimeter brightness profiles to find the best-fit parameters for each source (see their Table C.1).

The WISH target list is biased towards bright protostars, which are likely to be in a relatively active phase of accretion. Nonetheless, for the purpose of exploring the chemical effects of episodic accretion, we treat all sources as if they are currently in a quiescent phase. Alternative assumptions of some or all sources currently experiencing a high accretion rate would produce a different set of chemical models, but would not alter the conclusions in Sect. 4.

Within the best-fit models from Kristensen et al. (2012), we simulate an accretion burst by changing the stellar luminosity and rerunning DUSTY to obtain a new temperature profile. All other parameters remain constant. Our goal is to study the chemical effects of a strong burst, such as might happen at most a few times during the embedded phase. Specifically, the burst luminosity for each source is set to be 100 times higher than the current luminosity; this is the burst intensity inferred for IRAS 15398 (Jørgensen et al. 2013). The simulated bursts last for $100 \mathrm{yr}$ (Vorobyov \& Basu 2005), although shorter durations down to at least $1 \mathrm{yr}$ do not affect our results. After each burst ends, we follow the quiescent chemistry for a maximum of $10^{5} \mathrm{yr}$ to cover the full range of potential timescales from, e.g., Scholz et al. (2013). The models are static and ignore any dynamical effects of the envelope collapsing and dissipating on typical timescales of a few $10^{5} \mathrm{yr}$ (Evans et al. 2009; Visser et al. 2011). 
R. Visser et al.: Chemical tracers of episodic accretion in low-mass protostars

Table 1. Observed integrated intensities $\left(W \equiv \int T_{\mathrm{mb}} \mathrm{d} v\right.$ in $\left.\mathrm{K} \mathrm{km} \mathrm{s}^{-1}\right)$ and half-power beam widths $(\theta \text { in } \operatorname{arcsec})^{a}$.

\begin{tabular}{|c|c|c|c|c|c|c|c|c|c|c|c|c|c|}
\hline \multirow[t]{2}{*}{ Source } & \multicolumn{2}{|c|}{$\mathrm{HCO}^{+} 6-5$} & \multicolumn{2}{|c|}{$\mathrm{H}^{13} \mathrm{CO}^{+} 1-0$} & \multicolumn{2}{|c|}{$\mathrm{H}^{13} \mathrm{CO}^{+} 3-2$} & \multicolumn{2}{|c|}{$\mathrm{H}^{13} \mathrm{CO}^{+} 4-3$} & \multicolumn{2}{|c|}{$\mathrm{N}_{2} \mathrm{H}^{+} 1-0^{b}$} & \multicolumn{2}{|c|}{$\mathrm{N}_{2} \mathrm{H}^{+} 6-5^{b}$} & \multirow[t]{2}{*}{ References } \\
\hline & $W$ & $\theta$ & $W$ & $\theta$ & W & $\theta$ & W & $\theta$ & $W$ & $\theta$ & $W$ & $\theta$ & \\
\hline L1448 MM & 1.37 & 43 & 2.0 & 43 & 1.9 & 19 & 0.92 & 21 & 12.48 & 27 & 0.19 & 41 & $1,2,3,4$ \\
\hline NGC 1333 IRAS2A & 1.36 & 43 & 1.8 & 43 & 2.1 & 19 & 2.7 & 14 & 14.2 & 40 & 0.41 & 41 & $1,2,5$ \\
\hline NGC 1333 IRAS4A & 2.29 & 43 & 2.3 & 43 & 1.4 & 19 & 0.86 & 21 & 15.9 & 40 & 0.78 & 41 & $1,2,5,6$ \\
\hline NGC 1333 IRAS4B & 1.88 & 43 & 2.1 & 43 & 0.57 & 19 & & & 13.5 & 40 & 0.64 & 41 & $1,2,5$ \\
\hline L1527 & 1.06 & 43 & 2.2 & 43 & 1.1 & 19 & 0.47 & 14 & 3.91 & 27 & $<0.030$ & 41 & $1,2,4$ \\
\hline Ced110 IRS4 & & & & & & & 0.38 & 18 & 4.8 & 54 & $\ldots$ & & 7,8 \\
\hline BHR71 & 0.95 & 43 & & & & & . & & 8.1 & 54 & $\ldots$ & & 1,8 \\
\hline IRAS 15398 & 0.75 & 43 & & & 0.52 & 28 & .. & & 9.4 & 54 & $\cdots$ & & 8,9 \\
\hline L483 MM & 1.25 & 43 & 1.5 & 43 & 1.7 & 19 & 1.2 & 14 & 14.05 & 27 & 0.29 & 41 & $1,2,4$ \\
\hline Ser SMM1 & 4.30 & 43 & 1.56 & $c$ & 5.8 & 19 & 3.1 & 21 & 28 & 27 & 1.48 & 41 & $1,5,8,10$ \\
\hline Ser SMM4 & 2.08 & 43 & 1.13 & $c$ & 2.9 & 19 & 1.2 & 21 & 49 & 27 & . & & $1,8,10$ \\
\hline Ser SMM3 & 1.74 & 43 & $<0.1$ & $c$ & 1.6 & 19 & 0.2 & 21 & 23 & 27 & $\cdots$ & & $1,8,10$ \\
\hline L723 MM & 0.77 & 43 & 0.61 & 43 & 0.94 & 19 & 0.70 & 14 & 5.70 & 27 & .. & & $1,2,4$ \\
\hline B335 & 0.63 & 43 & 0.24 & 24 & 0.46 & 28 & . & & 6.05 & 27 & $\ldots$ & & $1,4,11$ \\
\hline L1157 & 0.54 & 43 & 0.89 & 29 & 0.61 & 19 & 0.52 & 14 & 8.38 & 27 & 0.07 & 41 & $1,2,4$ \\
\hline L1489 & 0.40 & 43 & 0.96 & 43 & 0.82 & 19 & 0.61 & 14 & 0.26 & 40 & $\ldots$ & & 2,5 \\
\hline L1551 IRS5 & . & & 1.55 & 19 & 2.4 & 19 & 2.2 & 14 & 15.7 & 40 & $\ldots$ & & 2,12 \\
\hline TMR1 & . & & 1.1 & 43 & 0.51 & 19 & 0.20 & 14 & 0.37 & 40 & $\ldots$ & & 2 \\
\hline TMC1A & . & & 0.44 & 19 & $<0.27$ & 19 & $<0.13$ & 14 & 7.1 & 40 & $\ldots$ & & 2,12 \\
\hline TMC1 & . & & 1.26 & 19 & $<0.27$ & 19 & $<0.12$ & 14 & 5.8 & 40 & $\ldots$ & & 2,12 \\
\hline HH46 IRS & . & & & & & & 1.1 & 18 & $\ldots$ & & $\ldots$ & & 7 \\
\hline Elias 29 & 0.28 & 43 & 0.36 & 72 & 0.09 & 28 & . & & $\ldots$ & & $\cdots$ & & 13 \\
\hline RNO91 & . & & 0.93 & 72 & . & & . & & 11 & 27 & $\cdots$ & & 8,14 \\
\hline
\end{tabular}

Notes. ${ }^{(a)}$ Throughout the paper, Class 0 sources appear above the horizontal line and Class I sources below it. $T_{\mathrm{mb}}$ is the main-beam temperature, corrected for beam efficiencies as detailed in the references. Typical uncertainties on the integrated intensities are 10-25\%. Upper limits are at the $3 \sigma$ level. See Yildiz et al. (2013) for a compilation of $\mathrm{C}^{18} \mathrm{O}$ intensities and beam sizes. ${ }^{(b)} \mathrm{The}_{2} \mathrm{H}^{+}$intensities are summed over all hyperfine components (see text). ${ }^{(c)}$ Integrated intensity over a 20" $\times 20^{\prime \prime}$ region (Hogerheijde et al. 1999).

References. (1) this work; (2) Jørgensen et al. (2004); (3) Gregersen et al. (1997); (4) Emprechtinger et al. (2009); (5) San José-García et al. (2015) and Benz et al. (2015); (6) Blake et al. (1995); (7) van Kempen et al. (2009); (8) Mardones et al. (1997); (9) Gregersen et al. (2000); (10) Hogerheijde et al. (1999); (11) Evans et al. (2005); (12) Onishi et al. (2002); (13) Boogert et al. (2002); (14) Butner et al. (1995).

Table 2. Observed ice column densities $\left(N(X)\right.$ in $\left.10^{17} \mathrm{~cm}^{-2}\right)$.

\begin{tabular}{lcccc}
\hline \hline Source & $\mathrm{CO}_{2}$ & $\mathrm{CO}$ & $\mathrm{H}_{2} \mathrm{O}$ & References \\
\hline L1527 & 10 & 18 & 47 & 1 \\
Ced110 IRS4 & 12.26 & 6.87 & $\ldots$ & 2 \\
IRAS 15398 & 52.16 & 8.35 & 148.0 & 2 \\
L723 MM & 49.0 & $\ldots$ & $\ldots$ & 3 \\
\hline L1489 & 16.20 & 7.13 & 47.0 & 2 \\
L1551 IRS5 & $11.86^{a}$ & $4.2^{b}$ & 109.0 & 4,5 \\
TMR1 & $4.92^{a}$ & $\ldots$ & 73.8 & 4 \\
TMC1A & $10.98^{a}$ & $\ldots$ & 53.1 & 4 \\
TMC1 & $12.71^{a}$ & $\ldots$ & 79.2 & 4 \\
HH46 IRS & 21.58 & 7.98 & 77.9 & 2 \\
GSS30 IRS1 & 3.28 & 2.53 & 15.3 & 2 \\
Elias 29 & 6.7 & 1.7 & 30.4 & 6,7 \\
Oph IRS63 & 6.84 & 3.49 & 20.4 & 2 \\
RNO91 & 11.66 & 7.46 & 39.0 & 2 \\
\hline
\end{tabular}

Notes. ${ }^{(a)}$ These values are scaled up by a factor of 1.36 relative to the published values from Zasowski et al. (2009), as recommended by Cook et al. (2011) to account for different $\mathrm{CO}_{2}$ laboratory band strengths. ${ }^{(b)}$ Scaled up by a factor of 1.4 to account for different band strengths.

References. (1) Aikawa et al. (2012); (2) Pontoppidan et al. (2008); (3) Cook et al. (2011); (4) Zasowski et al. (2009); (5) Tielens et al. (1991); (6) Boogert et al. (2000); (7) Boogert et al. (2008).

The heating and cooling rates of the dust are fast enough for our purposes that the dust temperature responds instantaneously to a change in luminosity (Johnstone et al. 2013). We set the gas temperature equal to the dust temperature at all times. As noted in Paper I, the temperature at any point in the envelope is proportional to $L_{*}^{0.25}$ and thus increases by a factor of 3.2 if the star becomes 100 times brighter (see also Johnstone et al. 2013). As an example, the top panel of Fig. 1 in Sect. 4 shows the "quiescent" and "burst" temperature profiles for IRAS 15398.

\subsection{Chemical network}

The basis of our chemical network is the RATE12 release of the UMIST Database for Astrochemistry (McElroy et al. 2013). In addition to standard neutral-neutral and ion-molecule chemistry, RATE12 includes photodissociation and photoionization reactions. These are important only at the outer edges of the envelopes, which we assume are irradiated by the mean interstellar radiation field. The cosmic-ray ionization rate is set to a standard value of $5 \times 10^{-17} \mathrm{~s}^{-1}$ (Dalgarno 2006). X-rays are not included; they have been detected in one $\mathrm{T}$ Tauri FUor (Liebhart et al. 2014), but X-ray luminosities in embedded protostars remain highly uncertain and are beyond the scope of this work.

We expand the network with freeze-out and evaporation of all neutral molecules. The freeze-out timescale depends inversely on the density and is typically of similar duration $\left(\sim 10^{3}-10^{4} \mathrm{yr}\right)$ as the quiescent phase between subsequent accretion bursts, allowing for an abundance pattern out of equilibrium with the stellar luminosity (Lee 2007; Paper I). Indeed, it is the long freeze-out timescale that drives most of the chemical aspects of episodic accretion explored in this work. 

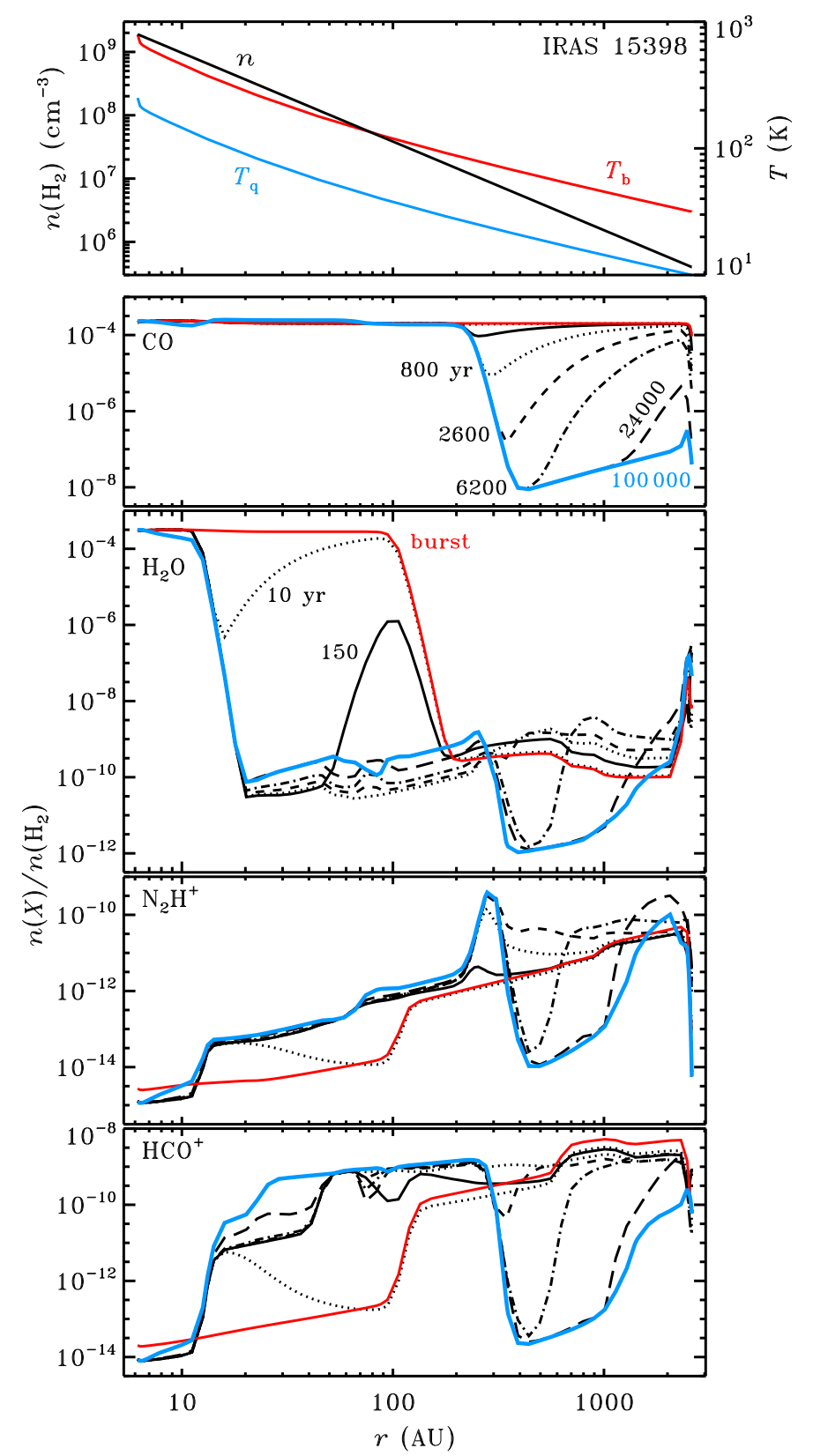

Fig. 1. Top panel: radial density profile in IRAS 15398 (black), along with the temperatures in the quiescent phase (blue) and during the burst (red). Other panels: radial abundance profiles of $\mathrm{CO}, \mathrm{H}_{2} \mathrm{O}, \mathrm{N}_{2} \mathrm{H}^{+}$, and $\mathrm{HCO}^{+}$. The red curves are during the accretion burst. The black curves are during the quiescent phase, at increasing amounts of time after the burst: $10 \mathrm{yr}$ (dotted), $150 \mathrm{yr}$ (solid), $800 \mathrm{yr}$ (dotted), $2600 \mathrm{yr}$ (shortdashed), $6200 \mathrm{yr}$ (dash-dotted), and $24000 \mathrm{yr}$ (long-dashed). The quiescent profiles culminate in the blue curve at $1 \times 10^{5} \mathrm{yr}$ after the burst.

Non-thermal desorption via cosmic rays (Hasegawa \& Herbst 1993) or UV radiation (Öberg et al. 2007) is included but only plays a minor role in episodic accretion chemistry. Much more important is thermal desorption. According to spectroscopic observations, protostellar CO evaporates at 25-30 K (Jørgensen et al. 2002, 2004, 2015; Y1ldiz et al. 2013). This is at least $5 \mathrm{~K}$ higher than predicted from binding energies measured in the laboratory, especially the "pure ice" value of $855 \mathrm{~K}$ from Bisschop et al. (2006) used in Paper I. Hence, we now adopt a binding energy of $1307 \mathrm{~K}$, measured for $\mathrm{CO}$ evaporating from amorphous water ice (Noble et al. 2012). Under protostellar conditions, this higher binding energy is consistent with the empirical evaporation temperature of $25-30 \mathrm{~K}$. Likewise, we increase the binding energy of $\mathrm{N}_{2}$ from $800 \mathrm{~K}$ to $1200 \mathrm{~K}$. The binding energy for atomic $\mathrm{O}$ has long been set to $800 \mathrm{~K}$ (Watson \& Salpeter 1972), but new experiments on water ice surfaces offer conclusive evidence that this old estimate is too low (Minissale 2014; Minissale et al., in prep.). We adopt the current best estimate of $1420 \mathrm{~K}$. The binding energies for other molecules are unchanged from Paper I, such as $5773 \mathrm{~K}$ for $\mathrm{H}_{2} \mathrm{O}$ and $2300 \mathrm{~K}$ for $\mathrm{CO}_{2}$ (Fraser et al. 2001; Noble et al. 2012).

Our network contains two types of grain-surface reactions. The first is the usual formation of $\mathrm{H}_{2}$ (Black \& van Dishoeck 1987). The second type is simple hydrogenation of $\mathrm{C}$ to $\mathrm{CH}_{4}, \mathrm{~N}$ to $\mathrm{NH}_{3}$, and $\mathrm{O}$ to $\mathrm{H}_{2} \mathrm{O}$. This happens one $\mathrm{H}$ atom at a time at a rate equal to the adsorption rate of $\mathrm{H}$ onto the grain surface multiplied by the relative fraction of the reactant molecule in the ice (Visser et al. 2011). Conversion of $\mathrm{CO}$ ice into $\mathrm{CO}_{2}$ ice, as proposed by Kim et al. $(2011,2012)$, is not included; see Sect. 5.1 for details.

The elemental abundances relative to the total number of $\mathrm{H}$ atoms are 0.09 for $\mathrm{He}, 1.4 \times 10^{-4}$ for $\mathrm{C}, 7.5 \times 10^{-5}$ for $\mathrm{N}$, and $3.2 \times 10^{-4}$ for O (McElroy et al. 2013). At the start of the first accretion burst, the chemical composition is set to typical prestellar core conditions (Maret et al. 2006; Whittet et al. 2009): hydrogen in atomic $\mathrm{H}(0.005 \%)$ and $\mathrm{H}_{2}(\sim 100 \%)$; carbon in $\mathrm{CO}$ (37\%), $\mathrm{CO}$ ice (35\%), and $\mathrm{CO}_{2}$ ice (28\%); remaining oxygen in $\mathrm{H}_{2} \mathrm{O}$ ice; and nitrogen in atomic $\mathrm{N}(55 \%), \mathrm{N}_{2}(42 \%)$, and $\mathrm{NH}_{3}$ ice $(3 \%)$. The initial ratio of $\mathrm{CO}_{2}$ ice to $\mathrm{CO}$ ice is $0.8: 1$, the average value observed in three dense molecular clouds believed to be representative of the earliest stage of star formation (Whittet et al. 2009).

\subsection{Line radiative transfer}

In order to compare our model to observations, we simulate molecular line spectra with the one-dimensional radiative transfer code RATRAN (Hogerheijde \& van der Tak 2000). RATRAN solves for the level populations as a function of position, accounting for both collisional and radiative excitation, and then performs ray tracing to synthesize a spectral image. Lastly, the images are convolved to the appropriate telescope beam for each source and transition (Table 1).

The low- $J$ lines of $\mathrm{CO}$ and $\mathrm{HCO}^{+}$are optically thick for most sources, so the optically thin isotopologs $\mathrm{C}^{18} \mathrm{O}$ and $\mathrm{H}^{13} \mathrm{CO}^{+}$are used instead. The isotope ratios are set to 69 for ${ }^{12} \mathrm{C} /{ }^{13} \mathrm{C}$ and 557 for ${ }^{16} \mathrm{O} /{ }^{18} \mathrm{O}$ (Wilson 1999). Collision rates are taken from Flower (1999), Daniel et al. (2005), and Yang et al. (2010), as compiled in the Leiden Atomic and Molecular Database ${ }^{1}$ (LAMDA; Schöier et al. 2005).

\section{Effects of accretion bursts on abundance profiles and rotational spectra}

As described in Sect. 3.1, we simulate an accretion burst in all 25 sources by increasing the luminosity to 100 times the observed luminosity for a duration of $100 \mathrm{yr}$. Starting with the prestellar core composition from Sect. 3.2, we evolve the chemistry during the burst at 78-89 radial points per source. After $100 \mathrm{yr}$, the burst ends and the temperature instantaneously returns to the quiescent profile. Continuing with the abundances from the end of the burst, we evolve the chemistry for another $10^{5} \mathrm{yr}$ and extract abundances at a number of intermediate time steps.

http://home.strw.leidenuniv.nl/ moldata 
All sources have the same qualitative abundance profiles and molecular line spectra, so we choose IRAS 15398 for a quantitative discussion. The results for the rest of the sample are presented in Sect. 5.

\subsection{Abundance profiles}

IRAS 15398 currently has a luminosity of $1.6 L_{\odot}$ and an envelope mass of $0.5 M_{\odot}$ (Kristensen et al. 2012). The top panel of Fig. 1 plots the temperature profiles for the quiescent phase $\left(T_{\mathrm{q}}\right)$ and the burst $\left(T_{\mathrm{b}}\right)$ in IRAS 15398 , along with the density profile $(n)$. The quiescent temperature decreases from $250 \mathrm{~K}$ at the inner edge of the envelope to $10 \mathrm{~K}$ at the outer edge. During the burst, the entire curve goes up by a factor of 3.2 (Sect. 3.1).

The other panels of Fig. 1 show the radial abundance profiles of $\mathrm{CO}, \mathrm{H}_{2} \mathrm{O}, \mathrm{N}_{2} \mathrm{H}^{+}$, and $\mathrm{HCO}^{+}$during the burst (red curves) and at a series of time steps after the burst (black and blue). The basic results for CO are the same as reported elsewhere (Lee 2007; Paper I; Vorobyov et al. 2013). The temperature exceeds $30 \mathrm{~K}$ everywhere during the burst and more than $99.9 \%$ of $\mathrm{CO}$ is in the gas phase at all radii ${ }^{2}$. After the burst, $\mathrm{CO}$ freezes out beyond $250 \mathrm{AU}$, where the quiescent temperature lies below $25 \mathrm{~K}$. Since the freeze-out rate is proportional to the gas density, depletion is fastest right around the $25 \mathrm{~K}$ radius. For the specific density profile in the envelope model of IRAS 15398, it takes $700 \mathrm{yr}$ for the $\mathrm{CO}$ abundance to drop by a factor of 10 and $1500 \mathrm{yr}$ for a factor of 100 .

The column density of CO ice integrated through the envelope is reduced almost to zero during the burst. Once the envelope cools down, the ice column starts to reform. It reaches $20 \%$ of the original amount after $100 \mathrm{yr}$ and $90 \%$ after $4000 \mathrm{yr}$. $\mathrm{CO}_{2}$ has a higher binding energy than $\mathrm{CO}$ and evaporates around $45 \mathrm{~K}$. This allows $15 \%$ of the integrated $\mathrm{CO}_{2}$ ice column to survive during the simulated burst in IRAS 15398. It takes $600 \mathrm{yr}$ in the quiescent phase to rebuild $90 \%$ of the original $\mathrm{CO}_{2}$ ice.

$\mathrm{H}_{2} \mathrm{O}$ evaporates out to $100 \mathrm{AU}$ during the burst. Like CO, the excess $\mathrm{H}_{2} \mathrm{O}$ refreezes in the quiescent phase, but it does so about ten times faster because of the higher densities in the inner envelope. All $\mathrm{H}_{2} \mathrm{O}$ that evaporated during the burst disappears within a few $100 \mathrm{yr}$ in the envelope model of IRAS 15398. On timescales of a few $1000 \mathrm{yr}$, the freeze-out of $\mathrm{CO}$ removes a substantial amount of oxygen from the gas phase. This leads to a drop in the $\mathrm{H}_{2} \mathrm{O}$ profile near the $25 \mathrm{~K}$ radius at $250 \mathrm{AU}$. The $\mathrm{H}_{2} \mathrm{O}$ abundance increases again towards the outer envelope because of photodesorption. The integrated column density of $\mathrm{H}_{2} \mathrm{O}$ ice drops by $65 \%$ during the burst; afterwards, it takes only $\sim 10 \mathrm{yr}$ to rebuild the column to $90 \%$ of the equilibrium value.

The chemistry of $\mathrm{N}_{2} \mathrm{H}^{+}$is strongly tied to that of $\mathrm{CO}$ and $\mathrm{H}_{2} \mathrm{O}$ because both act as important destruction channels. The depletion of $\mathrm{CO}$ and $\mathrm{H}_{2} \mathrm{O}$ in the quiescent phase thus allows $\mathrm{N}_{2} \mathrm{H}^{+}$to increase in abundance at almost all radii for the first few $1000 \mathrm{yr}$ after the burst. This type of anti-correlation between $\mathrm{CO}$ and $\mathrm{N}_{2} \mathrm{H}^{+}$is well known from observations of molecular clouds, embedded protostars, and circumstellar disks (Bergin et al. 2002; Jørgensen 2004; Qi et al. 2013) and was also noted by Lee (2007). On timescales of more than a few $1000 \mathrm{yr}$, freeze-out of $\mathrm{N}_{2}$ near $400 \mathrm{AU}$ causes a decrease in the $\mathrm{N}_{2} \mathrm{H}^{+}$abundance.

Lastly, the abundance of $\mathrm{HCO}^{+}$undergoes a seesaw motion around the $25 \mathrm{~K}$ radius. $\mathrm{CO}$ is the primary parent species of $\mathrm{HCO}^{+}$and $\mathrm{H}_{2} \mathrm{O}$ is one of the dominant destroyers. Freeze-out of $\mathrm{H}_{2} \mathrm{O}$ allows more $\mathrm{HCO}^{+}$to be formed in the inner envelope,

\footnotetext{
2 Lee (2007) simulated a lower-luminosity protostar and retained some $\mathrm{CO}$ ice in the outer envelope during the burst.
}

while freeze-out of $\mathrm{CO}$ reduces the amount of $\mathrm{HCO}^{+}$in the outer envelope on longer timescales. The correlation between the protostellar abundances of $\mathrm{CO}$ and $\mathrm{HCO}^{+}$is another wellestablished observational result (Jørgensen et al. 2004).

The chemical evolution in the other 24 envelope models is qualitatively the same as in IRAS 15398, but the timescales on which the aforementioned changes occur differ depending on the density profiles. IRAS 15398 has relatively high densities in the $\mathrm{H}_{2} \mathrm{O}$ and $\mathrm{CO}$ freeze-out zones compared to the rest of the sample. As a consequence, freeze-out in the other sources is typically slower by up to two orders of magnitude and the chemical effects of the accretion burst take longer to subside.

In summary, the chemical composition of a protostellar envelope changes substantially during an accretion burst. These changes last for long after the system returns to quiescence:

- enhanced $\mathrm{CO}$ in the outer envelope for a few $10^{3}-10^{4} \mathrm{yr}$;

- reduced $\mathrm{CO}$ ice and $\mathrm{CO}_{2}$ ice in the outer envelope for a few $10^{3}-10^{4} \mathrm{yr}$

- enhanced $\mathrm{H}_{2} \mathrm{O}$ in the inner envelope for a few $10^{2}-10^{3} \mathrm{yr}$;

- reduced $\mathrm{N}_{2} \mathrm{H}^{+}$in the outer envelope for a few $10^{3}-10^{4} \mathrm{yr}$;

- seesaw pattern for $\mathrm{HCO}^{+}$: enhanced in the inner envelope, reduced in the outer envelope for a few $10^{3}-10^{4} \mathrm{yr}$.

The exact timescales in a given source depend on the density profile. The next section explores the consequences of the abundance changes on the rotational emission lines.

\subsection{Rotational spectra and ice column densities}

The abundance changes caused by an accretion burst persist for long after the burst has ended. To what extent can the chemical effects of a burst still be observed once the protostar has returned to quiescence? Figure 2 shows a set of nine single-dish spectra corresponding to the abundance profiles at the quiescent time steps from Fig. 1. All spectra are first convolved with the appropriate telescope beam (Table 1) and then continuum-subtracted. The arrow in each panel indicates schematically how the peak intensity changes with time: continuous decrease, continuous increase, or an increase followed by a decrease.

For a more quantitative view of the temporal behavior, the spectra from Fig. 2 are integrated and plotted as a function of time in Fig. 3. Also shown here are the simulated column densities of $\mathrm{CO}_{2}, \mathrm{CO}$, and $\mathrm{H}_{2} \mathrm{O}$ ice. Where available, the observed intensities and column densities from Sect. 2 are included in Fig. 3 as gray bars with $20 \%$ error margins. We compare the model results to the observations in Sect. 5.1; the remainder of the current section discusses the model trends on their own.

During the quiescent phase, the simulated line intensities change predictably based on the abundance variations. The freeze-out of $\mathrm{CO}$ leads to a monotonic decrease in the optically thin $\mathrm{C}^{18} \mathrm{O}$ lines. $\mathrm{H}^{13} \mathrm{CO}^{+} 1-0$ and 3-2 are dominated by the cold outer envelope and their intensities also decrease monotonically as $\mathrm{CO}$ and $\mathrm{HCO}^{+}$are depleted. $\mathrm{H}^{13} \mathrm{CO}^{+} 4-3$ and $\mathrm{HCO}^{+} 6-5$ originate closer to the star and pick up on the seesaw abundance pattern from Fig. 1: the line intensities show an initial rise before depletion in the outer envelope sets in and the intensities go down.

Because of the abundance anticorrelation between $\mathrm{CO}$ and $\mathrm{N}_{2} \mathrm{H}^{+}$, the line intensities of $\mathrm{C}^{18} \mathrm{O}$ and $\mathrm{N}_{2} \mathrm{H}^{+}$evolve largely in opposite directions. As $\mathrm{CO}$ and $\mathrm{H}_{2} \mathrm{O}$ freeze out after the burst, $\mathrm{N}_{2} \mathrm{H}^{+}$becomes more abundant and its $1-0$ and 6-5 lines gain intensity. The 1-0 intensity decreases again on timescales of more than $24000 \mathrm{yr}$ after the burst. This is due to the depletion of 


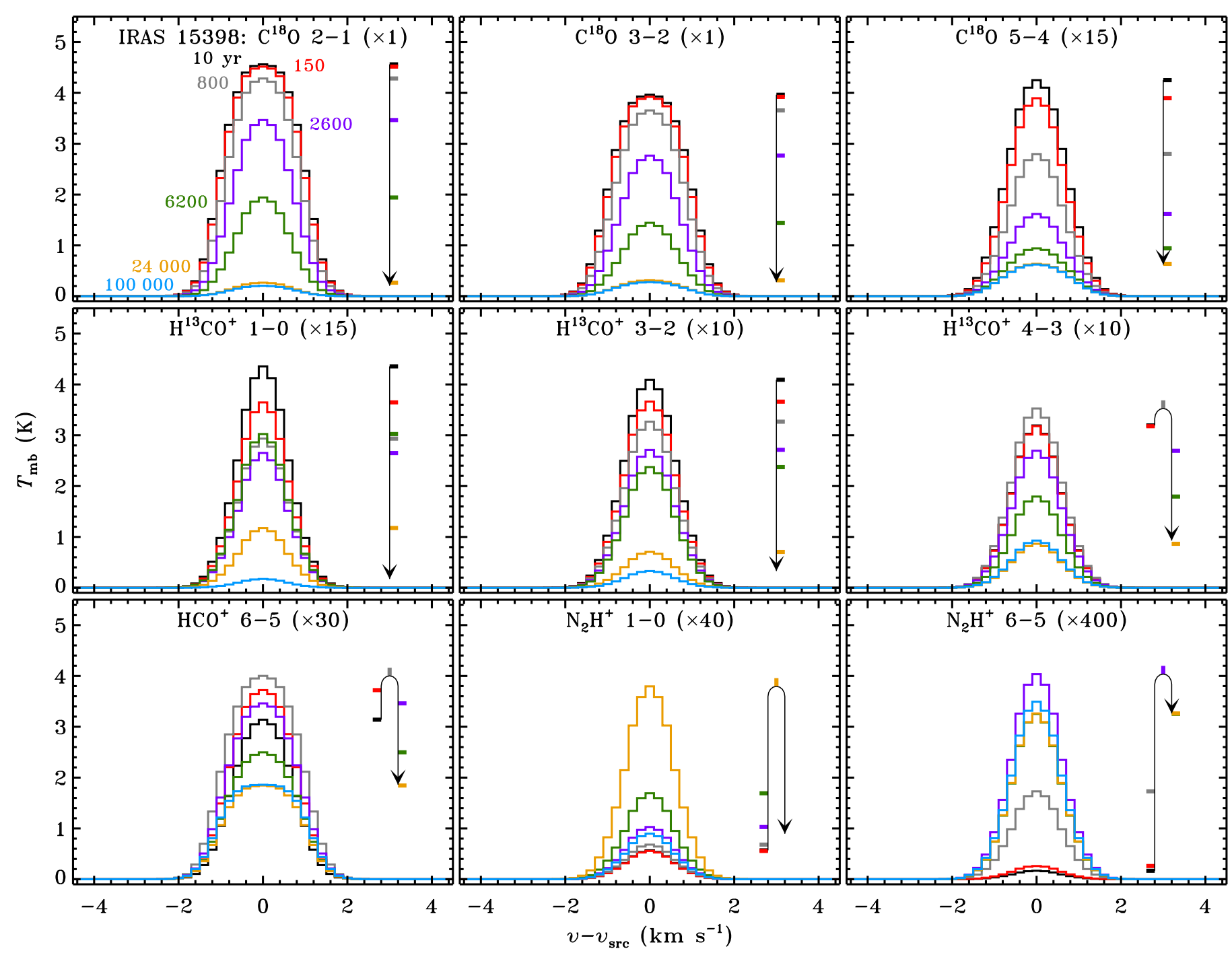

Fig. 2. Synthetic spectra for the quiescent phase in IRAS 15398, scaled as indicated. The different colors correspond to the same post-burst time steps as in Fig. 1: $10 \mathrm{yr}$ (black), $150 \mathrm{yr}$ (red), $800 \mathrm{yr}$ (gray), $2600 \mathrm{yr}$ (purple), $6200 \mathrm{yr}$ (green), $24000 \mathrm{yr}$ (orange), and $1 \times 10^{5} \mathrm{yr}$ (blue). The arrows indicate how the intensity of each line changes as a function of time; the colored flags mark the peak intensities at the listed times. The $\mathrm{N}_{2} \mathrm{H}^{+}$ 1-0 panel shows the isolated $F_{1}=0-1$ hyperfine component, whereas the 6-5 panel shows the sum over all unresolved components.

atomic $\mathrm{N}$ and the resulting loss of $\mathrm{N}_{2} \mathrm{H}^{+}$in the cold outer envelope. The 6-5 line is not very sensitive to the $\mathrm{N}_{2} \mathrm{H}^{+}$abundance around $1000 \mathrm{AU}$; its intensity levels off at late times, but does not turn over.

The total observable column densities of $\mathrm{CO}_{2}$ and $\mathrm{CO}$ ice increase by factors of 3 and 20 on timescales of $10^{3}-10^{4} \mathrm{yr}$ after the burst. The column of $\mathrm{H}_{2} \mathrm{O}$ ice is only marginally affected by changes in luminosity and remains constant at all times.

\section{Line ratios as a diagnostic of episodic accretion timescales}

One of the challenges in episodic accretion is to understand the magnitude-frequency distribution: how often do burst of a certain magnitude or intensity occur? The chemical effects explored in this work can help constrain the frequency of very strong bursts, where the accretion rate increases by at least a factor of 100. In the quiescent phase following such a burst, several observables vary monotonically with time. In principle, any of these can be used as a chronometer of when the most recent burst occurred. For a statistical sample of protostars, it then becomes possible to determine the average burst frequency.

With the current set of spherical models, inferring when the most recent burst occurred in any given source will be a rather crude method with uncertainties at the order-of-magnitude level. The following discussion should therefore be seen as an attempt to explore certain methodologies, rather than an attempt to derive firm numbers. For an alternative approach based on spatially resolved observations of $\mathrm{C}^{18} \mathrm{O}$, see Jørgensen et al. (2015).

\subsection{Models vs. observations}

Figure 3 highlights both the successes and the shortcomings of our model for IRAS 15398. Six of the observables are reproduced at some point during the quiescent phase, but the times at which the matches occur range over more than two orders of magnitude: from $\sim 30 \mathrm{yr}$ for $\mathrm{CO}$ ice to $\sim 10^{4} \mathrm{yr}$ for $\mathrm{C}^{18} \mathrm{O}$ $2-1$. Furthermore, $\mathrm{H}_{2} \mathrm{O}$ ice is overproduced at all times, and $\mathrm{HCO}^{+} 6-5$ and $\mathrm{N}_{2} \mathrm{H}^{+} 1-0$ are underproduced.

The overproduction of $\mathrm{H}_{2} \mathrm{O}$ ice may be due to a lack of grainsurface reactions in our chemical network, which tend to drain 
R. Visser et al.: Chemical tracers of episodic accretion in low-mass protostars

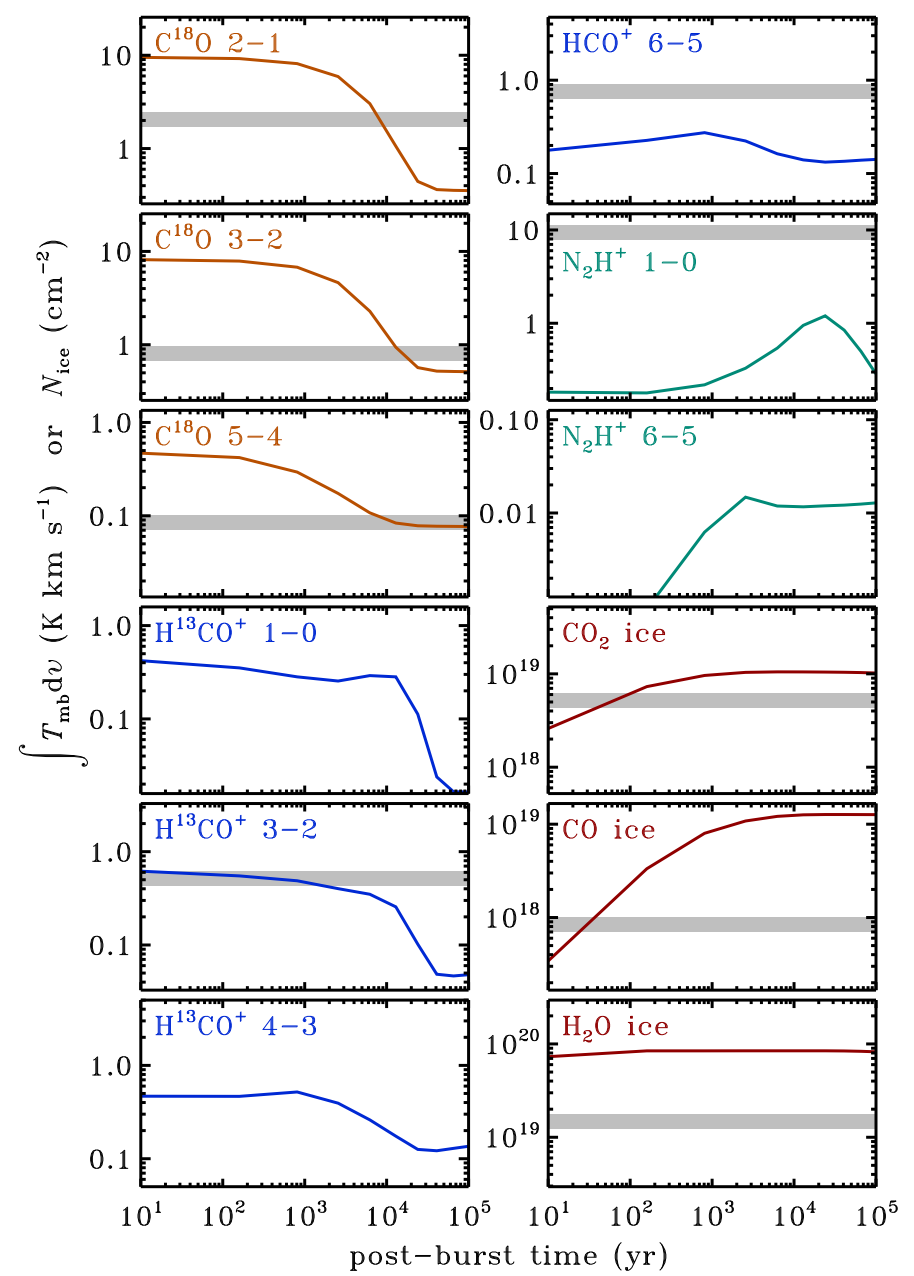

Fig. 3. Simulated integrated line intensities and ice column densities in IRAS 15398 as a function of time during the quiescent phase. The $\mathrm{N}_{2} \mathrm{H}^{+} J=1-0$ and 6-5 intensities are summed over all HF components. Where available, the gray bars show the observed intensities and columns from Tables 1 and 2 with $20 \%$ uncertainties.

oxygen out of $\mathrm{H}_{2} \mathrm{O}$ and convert it to other species (Schmalzl et al. 2014). $\mathrm{N}_{2} \mathrm{H}^{+} 1-0$ probably suffers from our choice of a spherically symmetric envelope model. In spatially resolved observations, the morphology of $\mathrm{N}_{2} \mathrm{H}^{+} 1-0$ often deviates significantly from spherical symmetry (Jørgensen 2004; Tobin et al. 2011). This can result in enhanced emission in one direction, e.g., from interactions between the outflow and the dense envelope, which our spherical models do not reproduce. The underproduction of $\mathrm{HCO}^{+} 6-5$ appears to be an excitation effect: this line is enhanced along the outflow cavity walls due to direct ultraviolet heating, similar to what is seen in ${ }^{12} \mathrm{CO}$ and ${ }^{13} \mathrm{CO} 6-5$ (Spaans et al. 1995; Y1ld1z et al. 2012).

Kim et al. $(2011,2012)$ argued that some fraction of CO ice needs to be converted into $\mathrm{CO}_{2}$ ice during each quiescent phase in between subsequent bursts. However, that conclusion may be an artifact of the low initial $\mathrm{CO}_{2}$ abundance in their model. Our model starts with the average $\mathrm{CO}$ and $\mathrm{CO}_{2}$ ice abundances observed towards dense cores (Whittet et al. 2009). As a result, it does not require any conversion of $\mathrm{CO}$ into $\mathrm{CO}_{2}$ ice to reproduce the observed column densities in IRAS 15398.

The comparison between model predictions and observations is expanded to all 25 protostars in Fig. 4. The trends identified in Figs. 2 and 3 for IRAS 15398 generally hold for the full sample: all $\mathrm{C}^{18} \mathrm{O}$ lines become weaker as a function of
Table 3. Candidate observable ratios to be used as chronometers of episodic accretion timescales.

\begin{tabular}{cc}
\hline \hline $\mathrm{C}^{18} \mathrm{O} 2-1 / \mathrm{N}_{2} \mathrm{H}^{+} 1-0$ & $\mathrm{C}^{18} \mathrm{O} 3-2 / \mathrm{N}_{2} \mathrm{H}^{+} 1-0$ \\
$\mathrm{H}^{13} \mathrm{CO}^{+} 1-0 / \mathrm{N}_{2} \mathrm{H}^{+} 1-0$ & $\mathrm{H}^{13} \mathrm{CO}^{+} 3-2 / \mathrm{N}_{2} \mathrm{H}^{+} 1-0$ \\
$\mathrm{C}^{18} \mathrm{O} 5-4 / \mathrm{N}_{2} \mathrm{H}^{+} 6-5$ \\
$\mathrm{C}^{18} \mathrm{O} 2-1 / \mathrm{CO}_{2}$ ice & $\mathrm{C}^{18} \mathrm{O} 2-1 / \mathrm{CO}$ ice \\
$\mathrm{C}^{18} \mathrm{O} 3-2 / \mathrm{CO}_{2}$ ice & $\mathrm{C}^{18} \mathrm{O}_{3}-2 / \mathrm{CO}$ ice \\
$\mathrm{H}^{13} \mathrm{CO}^{+} 1-0 / \mathrm{CO}_{2}$ ice & $\mathrm{H}^{13} \mathrm{CO}^{+} 1-0 / \mathrm{CO}$ ice \\
$\mathrm{H}^{13} \mathrm{CO}^{+} 3-2 / \mathrm{CO}_{2}$ ice & $\mathrm{H}^{13} \mathrm{CO}^{+} 3-2 / \mathrm{CO}$ ice \\
\hline
\end{tabular}

time and usually match the observed intensities at some point. $\mathrm{H}^{13} \mathrm{CO}^{+} 1-0$ and 3-2 also decrease monotonically and are in reasonable agreement with the observations. $\mathrm{H}^{13} \mathrm{CO}^{+} 4-3$ and $\mathrm{HCO}^{+} 6-5$ reflect the seesaw abundance pattern of $\mathrm{HCO}^{+}$by first increasing and then decreasing in strength. Both lines are too weak in the models by a factor of $2-10$. The $\mathrm{N}_{2} \mathrm{H}^{+} 1-0$ line gains intensity during the quiescent phase in all sources and turns over at late times in about a third of the sample. $\mathrm{N}_{2} \mathrm{H}^{+} 6-5$ initially increases and then levels off. Both $\mathrm{N}_{2} \mathrm{H}^{+}$lines tend to be underproduced relative to the observed intensities. The three ice column densities always increase with time after the burst. $\mathrm{CO}_{2}$ ice and $\mathrm{CO}$ ice usually match the observations, but $\mathrm{H}_{2} \mathrm{O}$ ice is consistently too abundant in the models.

In order to construct a consistent set of models, we treated all sources as if they are currently in the quiescent phase (Sect. 3.1). This is undoubtedly a false assumption - especially for the brighter sources in our target list - and adds another source of discrepancies between models and observations. Future studies of individual sources or large samples will benefit from independent constraints on the accretion rates, e.g., from hydrogen $\operatorname{Br} \gamma$ (Connelley \& Greene 2010). If the stellar mass is known from measurements of a rotationally supported disk (Tobin et al. 2012), the accretion rate can also be computed from the relationship between $L_{\mathrm{bol}}, M_{*}$, and $\dot{M}$ (Adams \& Shu 1986).

\subsection{Line ratios}

The absolute line intensities in Fig. 3 are sensitive to uncertainties in the source distance and in various model parameters like the envelope mass and the temperature profile. A better approach is to take the ratio between two rotational lines whose intensities change in opposite directions after the burst. This effectively acts as a self-normalization against distance and source model, in particular for line pairs at similar excitation levels.

Table 3 lists thirteen observable ratios as candidate chronometers, chosen in part for their sensitivity to the burst, and in part for being easily observable with modern submillimeter facilities. The first four ratios are the low- $J$ line pairs of $\mathrm{C}^{18} \mathrm{O} 2-1$, $\mathrm{C}^{18} \mathrm{O} 3-2, \mathrm{H}^{13} \mathrm{CO}^{+} 1-0$, and $\mathrm{H}^{13} \mathrm{CO}^{+} 3-2$ over $\mathrm{N}_{2} \mathrm{H}^{+} 1-0$. The fifth one is the high- $J$ pair of $\mathrm{C}^{18} \mathrm{O} 5-4$ over $\mathrm{N}_{2} \mathrm{H}^{+} 6-5$. The remaining eight are the ratios between the intensities of $\mathrm{C}^{18} \mathrm{O} 2-1$, $\mathrm{C}^{18} \mathrm{O} 3-2, \mathrm{H}^{13} \mathrm{CO}^{+} 1-0$, and $\mathrm{H}^{13} \mathrm{CO}^{+} 3-2$ on the one hand and the column densities of $\mathrm{CO}_{2}$ or $\mathrm{CO}$ ice on the other hand.

As an example, the left panel of Fig. 5 shows how the ratio between the integrated intensity of $\mathrm{C}^{18} \mathrm{O} 2-1$ and the column density of $\mathrm{CO}$ ice evolves as a function of time after the burst for the full source sample. The ratio plots for the other 12 pairs of observables in Table 3 look qualitatively the same. Each curve represents one source model. The ratios decreases with time for all sources, but the curves are scattered across more than four orders of magnitude and are of little diagnostic value in this form. 


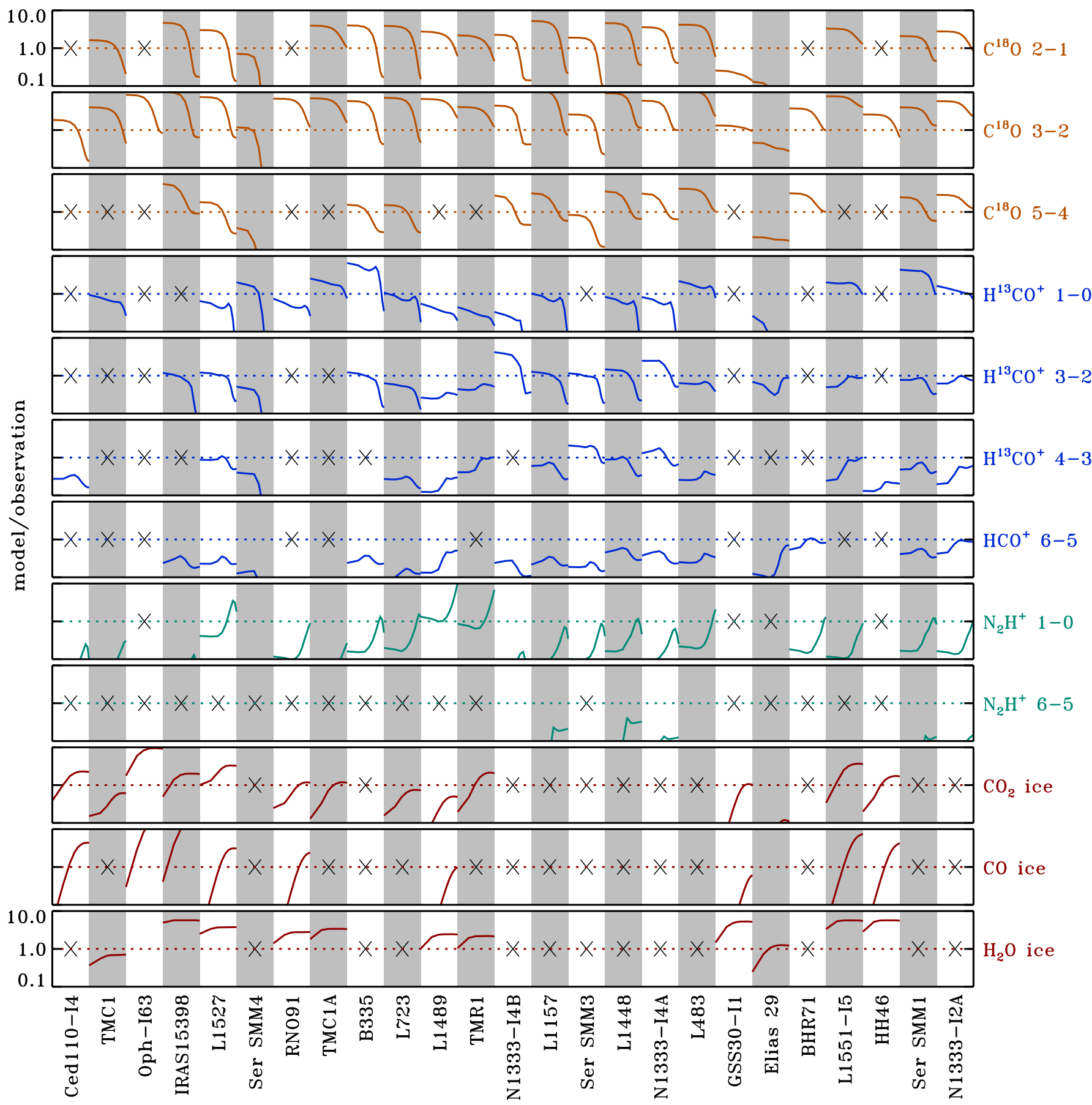

Fig. 4. Comparison between model predictions and observations. This plot is similar to Fig. 3, except that the model values are now divided by the observed quantities. A ratio of unity means the model is in exact agreement with observations. If the ratio is larger than unity, the model overpredicts the observation, and vice versa. The horizontal scale for each source runs logarithmically from 10 to $10^{5} \mathrm{yr}$ after the burst. The observable quantity in the bottom three panels is the column density of ice along a pencil beam to the central star. In the other panels, it is the integrated intensity of various rotational lines. The $\mathrm{N}_{2} \mathrm{H}^{+} J=1-0$ and 6-5 intensities are summed over all HF components. The sources are ordered from left to right by increasing luminosity.

The scatter in Fig. 5 is due to the range of envelope parameters encountered across the sample. CO freezes out at the same temperature in each source $(<25 \mathrm{~K})$, but because of all the different envelope parameters, it does not do so at the same density. In high-density envelopes, the freeze-out timescale is shorter and the $\mathrm{CO}$ gas/ice abundance ratio decreases more rapidly than in lower-density sources. Likewise, the $\mathrm{C}^{18} \mathrm{O} / \mathrm{N}_{2} \mathrm{H}^{+}$ and $\mathrm{H}^{13} \mathrm{CO}^{+} / \mathrm{N}_{2} \mathrm{H}^{+}$line intensity ratios change at different rates for different sources.
The scatter in the left panel of Fig. 5 can be reduced by expressing the time axis for each source in units of its characteristic freeze-out timescale (Paper I, erratum):

$\tau_{\mathrm{fr}}=1 \times 10^{4} \mathrm{yr} \sqrt{\frac{10 \mathrm{~K}}{T_{\mathrm{fr}}}} \frac{10^{6} \mathrm{~cm}^{-3}}{n_{\mathrm{fr}}}$.

The characteristic freeze-out density $n_{\mathrm{fr}}$ is defined as the density at which the temperature reaches $T_{\mathrm{fr}}$ in the quiescent phase. We 
R. Visser et al.: Chemical tracers of episodic accretion in low-mass protostars
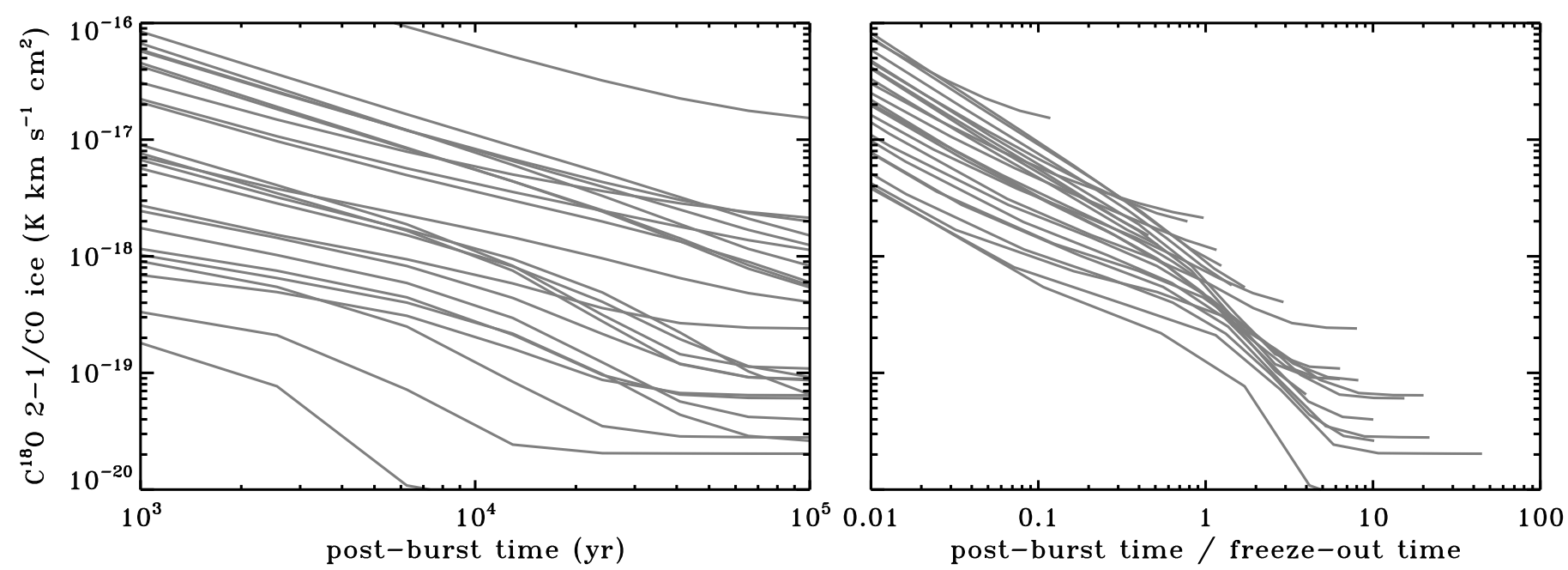

Fig. 5. Left: ratio between the integrated intensity of $\mathrm{C}^{18} \mathrm{O} 2-1$ and the column density of $\mathrm{CO}$ ice as a function of time during the quiescent phase. Each gray curve corresponds to one source. Right: same, but with the time axis normalized by the characteristic freeze-out timescale for each source.

tested $T_{\text {fr }}$ from 10 to $40 \mathrm{~K}$ and found the greatest reduction in scatter for $15 \mathrm{~K}$, where the bulk of the low- $J \mathrm{C}^{18} \mathrm{O}$ emission originates. At $15 \mathrm{~K}, n_{\mathrm{fr}}$ ranges from $3.5 \times 10^{3} \mathrm{~cm}^{-3}$ in Elias 29 to $5.5 \times 10^{6} \mathrm{~cm}^{-3}$ in Ser SMM4. The corresponding freeze-out timescales vary from 2.3 Myr to $1500 \mathrm{yr}$.

The time-normalized $\mathrm{C}^{18} \mathrm{O} 2-1 / \mathrm{CO}$ ice ratios appear as the gray curves in the right panel of Fig. 5. The sources are still scattered across an order of magnitude, but all follow the same trend and an average relationship between normalized post-burst time and gas/ice observable ratio can easily be drawn. Our source sample is probably diverse enough (albeit not unbiased) that we can use this average relationship also for other samples where detailed envelope models are not readily available.

\subsection{Future prospects}

Using all the observable ratios from Table 3, we can estimate when the most recent accretion burst occurred in each of our sources. However, the results are currently too scattered and the uncertainties too large to produce reliable numbers worth tabulating. For example, Jørgensen et al. (2013) concluded from spatially resolved $\mathrm{H}^{13} \mathrm{CO}^{+} 4-3$ that IRAS 15398 experienced a burst between 100 and $1000 \mathrm{yr}$ ago. We find the same timescale from the $\mathrm{C}^{18} \mathrm{O}$ gas $/ \mathrm{CO}_{2}$ ice ratios, but $\mathrm{C}^{18} \mathrm{O}$ gas/CO ice suggests a longer timescale of $\sim 6000 \mathrm{yr}$, whereas $\mathrm{H}^{13} \mathrm{CO}^{+} 3-2 / \mathrm{N}_{2} \mathrm{H}^{+} 1-0$ yields a value as long as $5 \times 10^{4} \mathrm{yr}$. Furthermore, the observed $\mathrm{C}^{18} \mathrm{O} / \mathrm{N}_{2} \mathrm{H}^{+}$line ratios do not match any timescale from the model. The situation is no better for most of the other sources. This lack of consistency between different tracers was already evident in Figs. 3 and 4, where some curves match the observations at very different post-burst times (Sect. 5.1).

The most likely reason for these discrepancies is the assumption of spherical symmetry for the envelope models (Sect. 5.1). However, even if the spherical models do not produce reliable timescales, they are still useful as a basis for follow-up studies. Spatially resolved observations offer many advantages over single-dish data (as explored for, e.g., $\mathrm{C}^{18} \mathrm{O} 2-1$ by Jørgensen et al. 2015) and can be carried out for statistically significant source samples with current facilities. We will expand the models beyond spherical symmetry and calibrate them against existing interferometric data to identify the most reliable tracers of episodic accretion timescales. These tracers can then be used to derive timescales for many tens or hundreds of protostars and compared against results from other methods. Working with such large samples also reduces the problem that any given source may be an outlier.

\section{Conclusions}

This paper simulates the chemical effects of episodic accretion in a diverse sample of 25 protostellar envelope models. The intensity of the accretion bursts is set to 100 times the quiescent luminosity, enough to evaporate $\mathrm{CO}$ ice all the way to the outer edge of the envelope in each source (Sect. 4.1). The $\mathrm{H}_{2} \mathrm{O}$ snowline expands in size by a factor of $\sim 10$ during the burst. The evaporation of $\mathrm{CO}$ and $\mathrm{H}_{2} \mathrm{O}$ leads to the destruction of $\mathrm{N}_{2} \mathrm{H}^{+}$ throughout the envelope. $\mathrm{HCO}^{+}$is enhanced outside of the $\mathrm{H}_{2} \mathrm{O}$ snowline and destroyed inside of it.

When the burst ends, the temperatures quickly return to normal. The species that evaporated during the burst start to refreeze onto the cold dust, but this is a relatively slow process. $\mathrm{H}_{2} \mathrm{O}$ remains enhanced for $10^{2}-10^{3} \mathrm{yr}$ and $\mathrm{CO}$ for an order of magnitude longer. The abundances of $\mathrm{N}_{2} \mathrm{H}^{+}$and $\mathrm{HCO}^{+}$are reset on similarly long timescales.

The abundance changes resulting from an accretion burst also have a substantial effect on various molecular lines, as quantified by radiative transfer simulations at a series of time steps (Sect. 4.2). $\mathrm{C}^{18} \mathrm{O} 2-1,3-2$, and 5-4 and $\mathrm{H}^{13} \mathrm{CO}^{+} 1-0$ and 3-2 are enhanced during the burst and decay monotonically after the burst ends. $\mathrm{H}^{13} \mathrm{CO}^{+} 4-3, \mathrm{HCO}^{+} 6-5$, and $\mathrm{N}_{2} \mathrm{H}^{+} 1-0$ first increase after the burst and then decrease. The column densities of $\mathrm{CO}$ and $\mathrm{CO}_{2}$ ice increase monotonically after the burst.

The simulated line intensities and ice column densities are compared to single-dish observations gathered from the literature and to previously unpublished spectra of $\mathrm{HCO}^{+}$and $\mathrm{N}_{2} \mathrm{H}^{+}$6-5 from the Herschel Space Observatory. Several line ratios are identified that can be used, in principle, as a chronometer of when the most recent strong burst occurred in each source (Sect. 5). In practice, however, the spherical source models are not accurate enough to derive reliable timescales from single-dish data. Interferometric observations are therefore recommended for follow-up work. In order to tackle a statistically significant source sample, the models need to be calibrated 
against spatially resolved observations to identify robust tracers of episodic accretion timescales.

Acknowledgements. This work was supported by the National Science Foundation under grant 1008800 and by a Postdoctoral Fellowship from the European Southern Observatory. J.K.J. was supported by a Lundbeck Foundation Junior Group Leader Fellowship and by the Centre for Star and Planet Formation, funded by the Danish National Research Foundation. This research made use of NASA's Astrophysics Data System and of the SIMBAD database, operated at CDS, Strasbourg, France.

\section{Appendix A: Details of observations}

The spectroscopic observations used in this work are summarized in Tables 1 and 2. The following sections offer more details on how the data were obtained and reduced. The $\mathrm{HCO}^{+}$and $\mathrm{N}_{2} \mathrm{H}^{+} 6-5$ spectra are published here for the first time; all other observations were gathered from the literature. Typical calibration uncertainties are between $10 \%$ and $25 \%$.

\section{A.1. $\mathrm{C}^{18} \mathrm{O}$}

Yildiz et al. (2013) compiled all available $\mathrm{C}^{18} \mathrm{O}$ data from $J=2-1$ up to $10-9$, with one omission: an integrated intensity of $11.0 \mathrm{~K} \mathrm{~km} \mathrm{~s}^{-1}$ in a $22.9^{\prime \prime}$ beam for the 2-1 line in Ser SMM4 (Hogerheijde et al. 1999). The 6-5 and higher lines are detected in only a handful of sources and are therefore of limited use as an observational diagnostic. The 2-1, 3-2, and 5-4 lines have detection rates of $77 \%, 100 \%$, and $58 \%$ and are well suited for our purpose. Furthermore, with upper-level energies of 16, 32, and $79 \mathrm{~K}$, these three lines together are a good probe of the abundance jump at the CO sublimation front (Y1ldiz et al. 2013).

\section{A.2. $\mathrm{HCO}^{+}$}

The low- $J$ lines of $\mathrm{HCO}^{+}$are often optically thick, so we only consider the $6-5$ line at $535 \mathrm{GHz}\left(E_{\mathrm{u}} / k=90 \mathrm{~K}\right)$. This transition was observed with Herschel-HIFI as part of WISH and the related open-time program "Water in low-mass protostars: the William Herschel line legacy" (WILL; proposal code OT2_evandish_4; Mottram et al., in prep.). Five of the spectra were presented by San José-García et al. (2015) and Benz et al. (2015). The other eleven $\mathrm{HCO}^{+}$lines from this data set are as yet unpublished.

Table A.1 lists the observing dates and identification numbers (ObsIDs), including several duplicate or triplicate observations taken in spectral setups of other lines. Five of these come from the open-time program "Searching for the onset of energetic particle irradiation in Class 0 protostars" (OT2_cceccare_4).

All observations were taken in double beam switch mode. We use the spectra recorded with the wide-band spectrometer (WBS; $1.1 \mathrm{MHz}$ resolution) and perform basic standard processing in HIPE v10.0.0 (Ott 2010), followed by further analysis in $\mathrm{CLASS}^{3}$. The $\mathrm{H}$ and $\mathrm{V}$ polarization spectra were averaged after individual inspection and the intensities were converted to mainbeam temperature scale through a main-beam efficiency of 0.72 (Roelfsema et al. 2012). Where available, the multiple epochs of data all match to within the calibration uncertainty of $10 \%$ and are averaged to reduce the noise. This results in final rms noise levels of $3-7 \mathrm{mK}$ in $0.28 \mathrm{~km} \mathrm{~s}^{-1}$ bins. The final data reduction step was to subtract a linear baseline.

\footnotetext{
3 http://wWw . iram. fr/IRAMFR/GILDAS
}

Table A.1. Herschel observations of $\mathrm{HCO}^{+}$and $\mathrm{N}_{2} \mathrm{H}^{+}$.

\begin{tabular}{|c|c|c|c|}
\hline Source & ObsID & Date & Ref. \\
\hline \multicolumn{4}{|c|}{$\mathrm{HCO}^{+} 6-5$ at $535.062 \mathrm{GHz}$} \\
\hline L1448 MM & 1342203186 & 2010 Aug. 18 & 1 \\
\hline \multirow[t]{3}{*}{ NGC 1333 IRAS2A } & 1342192206 & 2010 Mar. 14 & 1 \\
\hline & 1342202024 & 2010 Aug. 1 & 1 \\
\hline & 1342248912 & 2012 Jul. 31 & 2 \\
\hline \multirow[t]{3}{*}{ NGC 1333 IRAS4A } & 1342192207 & 2010 Mar. 15 & 1 \\
\hline & 1342202023 & 2010 Aug. 1 & 1 \\
\hline & 1342248914 & 2012 Jul. 31 & 2 \\
\hline \multirow[t]{3}{*}{ NGC 1333 IRAS4B } & 1342192208 & 2010 Mar. 15 & 1 \\
\hline & 1342202022 & 2010 Aug. 1 & 1 \\
\hline & 1342202033 & 2010 Aug. 2 & 1 \\
\hline \multirow[t]{2}{*}{ L1527 } & 1342203188 & 2010 Aug. 18 & 1 \\
\hline & 1342250193 & 2012 Aug. 24 & 2 \\
\hline BHR71 & 1342200755 & $2010 \mathrm{Jul} .7$ & 1 \\
\hline IRAS 15398 & 1342266008 & 2013 Mar. 5 & 3 \\
\hline L483 MM & 1342207582 & 2010 Oct. 28 & 1 \\
\hline \multirow[t]{3}{*}{ Ser SMM1 } & 1342194463 & 2010 Apr. 11 & 1 \\
\hline & 1342207581 & 2010 Oct. 28 & 1 \\
\hline & 1342251637 & 2012 Sep. 29 & 2 \\
\hline Ser SMM4 & 1342194464 & 2010 Apr. 11 & 1 \\
\hline Ser SMM3 & 1342207580 & 2010 Oct. 28 & 1 \\
\hline L723 MM & 1342219172 & 2011 Apr. 21 & 1 \\
\hline B335 & 1342219182 & 2011 Apr. 21 & 1 \\
\hline \multirow[t]{2}{*}{ L1157 } & 1342199077 & 2010 Jun. 23 & 1 \\
\hline & 1342246462 & 2012 May 17 & 2 \\
\hline L1489 & 1342203187 & 2010 Aug. 18 & 1 \\
\hline Elias 29 & 1342266143 & 2013 Mar. 7 & 1 \\
\hline \multicolumn{4}{|c|}{$\mathrm{N}_{2} \mathrm{H}^{+} 6-5$ at $558.967 \mathrm{GHz}$} \\
\hline L1448 MM & 1342238644 & 2012 Feb. 3 & 4 \\
\hline \multirow[t]{2}{*}{ NGC 1333 IRAS2A } & 1342225935 & 2011 Aug. 9 & 4 \\
\hline & 1342248913 & 2012 Jul. 31 & 2 \\
\hline \multirow[t]{2}{*}{ NGC 1333 IRAS4A } & 1342238646 & 2012 Feb. 3 & 4 \\
\hline & 1342248915 & 2012 Jul. 31 & 2 \\
\hline NGC 1333 IRAS4B & 1342238645 & 2012 Feb. 3 & 4 \\
\hline L1527 & 1342250194 & 2012 Aug. 24 & 2 \\
\hline L483 MM & 1342244045 & 2012 Apr. 10 & 4 \\
\hline \multirow[t]{2}{*}{ Ser SMM1 } & 1342230370 & 2011 Oct. 8 & 4 \\
\hline & 1342251636 & 2012 Sep. 29 & 2 \\
\hline L1157 & 1342246461 & $2012-05-17$ & 2 \\
\hline
\end{tabular}

References. (1) WISH: San José-García et al. (2015), Benz et al. (2015); (2) OT2_cceccare_4; (3) OT2_evandish_4: Mottram et al., in prep.; (4) OT1_philybla_1.

In all but two sources, the $\mathrm{HCO}^{+} 6-5$ spectrum shows two emission features: a broad component with a full width at half maximum (FWHM) of 4-14 $\mathrm{km} \mathrm{s}^{-1}$ and a narrow component of $1-2 \mathrm{~km} \mathrm{~s}^{-1}$. These components are also detected in various $\mathrm{CO}$ lines and correspond to the outflow (broad) and the quiescent envelope (narrow; Y1ldız et al. 2013; San José-García et al. 2015). The two exceptions in our sample are L723 and B335, which only have the narrow component.

Since we are interested exclusively in the quiescent envelope, we fit two Gaussian profiles to each $\mathrm{HCO}^{+}$spectrum (except L723 and B335) and subtract the outflow emission. Figure A.1 shows the resulting spectra, corrected for the source velocities from Yildiz et al. (2012). Table 1 lists the integrated intensities for the full sample.

\section{A.3. $\mathrm{H}^{13} \mathrm{CO}^{+}$}

Complementing the $\mathrm{HCO}^{+} 6-5$ line are the 1-0, 3-2, and 4-3 lines of optically thin $\mathrm{H}^{13} \mathrm{CO}^{+}$. Their upper-level energies 
R. Visser et al.: Chemical tracers of episodic accretion in low-mass protostars

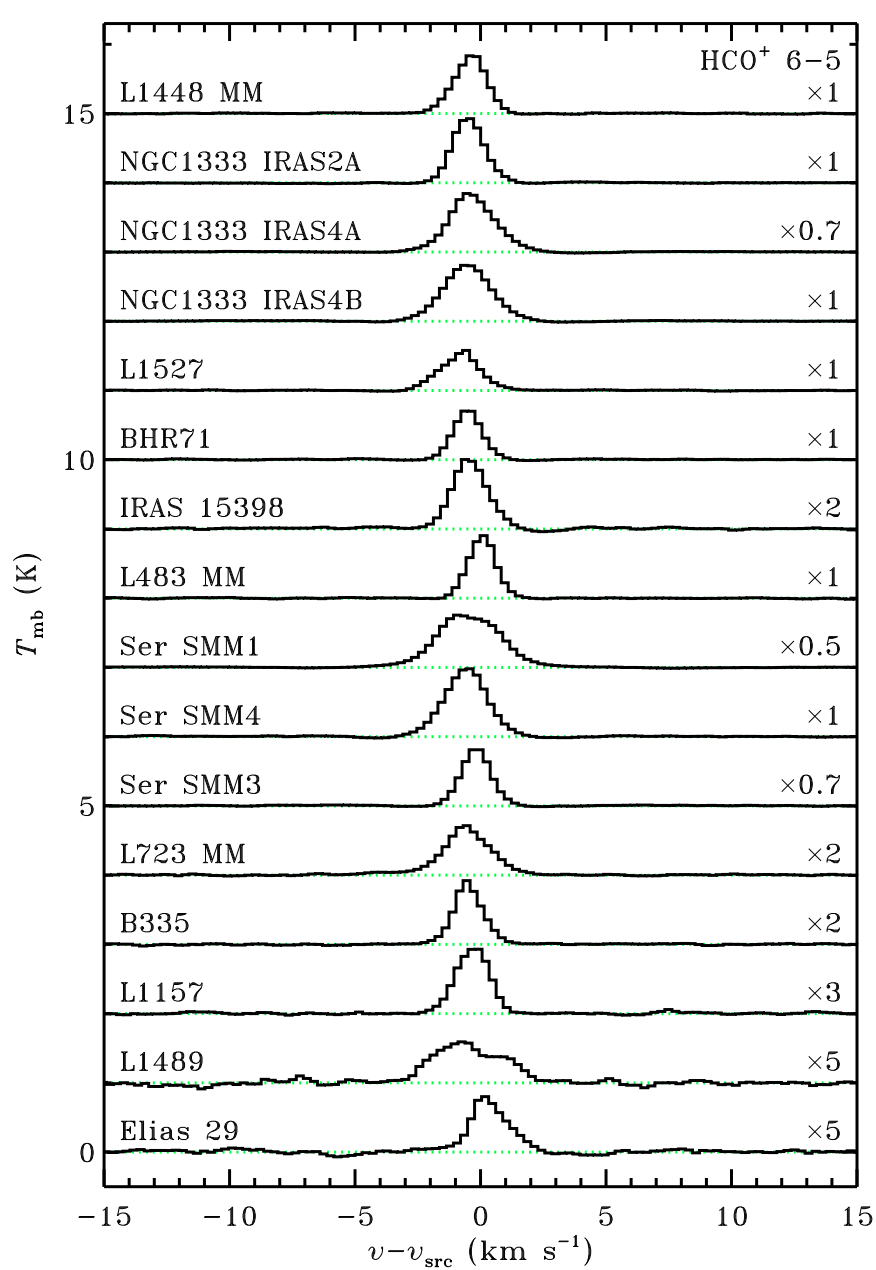

Fig. A.1. Spectra of $\mathrm{HCO}^{+} 6-5$, corrected for source velocity and scaled as indicated. If a broad emission component from the outflow was present (in all sources but L723 and B335), it was fitted with a Gaussian profile and subtracted from the spectrum to leave only the narrow emission from the quiescent envelope.

are 4,25 , and $42 \mathrm{~K}$, again offering a good probe of chemical changes induced by the evaporation or freeze-out of CO.

The integrated intensities compiled in Table 1 originate mostly from the ground-based surveys of Hogerheijde et al. (1999) and Jørgensen et al. (2004), with other references as indicated. Some sources have been targeted with multiple telescopes, in which case we choose the observation with the smallest single-dish beam.

\section{A.4. $\mathrm{N}_{2} \mathrm{H}^{+}$}

The final two molecular lines are the $J=1-0$ and 6-5 transitions of $\mathrm{N}_{2} \mathrm{H}^{+}\left(E_{\mathrm{u}} / k=4\right.$ and $\left.94 \mathrm{~K}\right)$, both of which are prone to hyperfine splitting (Caselli et al. 1995). The quantum numbers for the HF levels are $F_{1}$ and $F$. The $1-0$ transition breaks up into one isolated line $\left(F_{1}=0-1\right)$ and two clusters of three lines $\left(F_{1}=1-1\right.$ and $\left.2-1\right)$, which are readily resolved with heterodyne spectrographs. The $6-5$ transition is dominated by nine HF components within $0.1 \mathrm{~km} \mathrm{~s}^{-1}$ and therefore appears as a single line in astronomical observations.

Most of the $J=1-0$ intensities in Table 1 are taken from Jørgensen et al. (2004) and Emprechtinger et al. (2009), who provided integrated intensities summed over all HF components. Additional data are taken from Mardones et al. (1997), who only

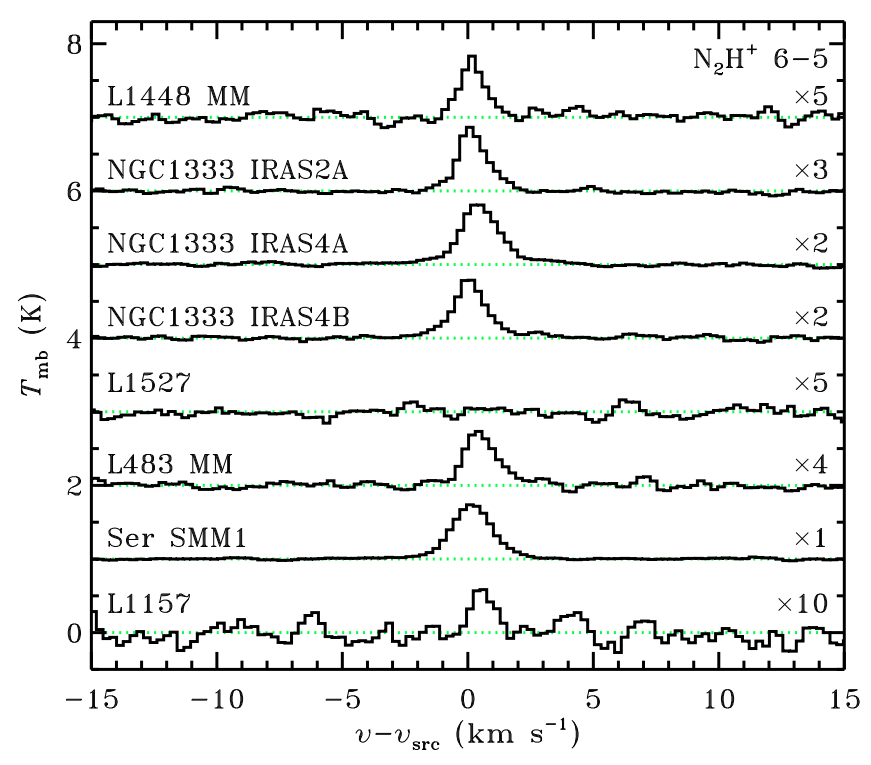

Fig. A.2. Spectra of $\mathrm{N}_{2} \mathrm{H}^{+} 6-5$, corrected for source velocity and scaled as indicated.

showed the spectra of the isolated component and did not tabulate any intensities. We measure the integrated intensities from their Figs. 1 and 2 and multiply by a factor of 9 (based on the Einstein A coefficients and level degeneracies) to approximate the intensity for the full 1-0 band.

The 6-5 lines were observed primarily as part of the Herschel open-time program "The chemistry of nitrogen in dark clouds" (OT1_philybla_1), with two additional sources (L1527 and L1157) covered by OT2_cceccare_4. Both programs targeted $\mathrm{N}_{2} \mathrm{H}^{+}$6-5 in NGC 1333 IRAS2A and 4A and Ser SMM1. None of these spectra have been published, so we follow the same procedure as outlined for $\mathrm{HCO}^{+} 6-5$ in Sect. A.2. Table A.1 lists the observing dates and ID codes and Fig. A.2 shows the reduced spectra. The rms noise is $10-14 \mathrm{mK}$ in $0.27 \mathrm{~km} \mathrm{~s}^{-1}$ bins. $\mathrm{N}_{2} \mathrm{H}^{+} 6-5$ is not detected in L1527. All other sources show a single narrow emission feature with an FWHM of $1-2 \mathrm{~km} \mathrm{~s}^{-1}$ and no contribution from the outflow. Table 1 lists the integrated intensities for the full sample, including the upper limit for L1527.

\section{A.5. Ices}

The ice column densities in Table 2 are based on published midinfrared observations from the ground and from space. The conversion from spectrum to column density requires an absorption band strength measured in the laboratory for the appropriate pure or mixed ices (Gerakines et al. 1995). Most papers on protostellar ices use the same band strengths and we adopt the column densities as published. The two exceptions are the $\mathrm{CO}_{2}$ columns of Zasowski et al. (2009) and the CO column of Tielens et al. (1991), which we scale by a common factor of 1.4 to be consistent with other studies.

\section{References}

Ábrahám, P., Juhász, A., Dullemond, C. P., et al. 2009, Nature, 459, 224

Adams, F. C., \& Shu, F. H. 1986, ApJ, 308, 836

Aikawa, Y., Kamuro, D., Sakon, I., et al. 2012, A\&A, 538, A57

Arce, H. G., Mardones, D., Corder, S. A., et al. 2013, ApJ, 774, 39

Audard, M., Ábrahám, P., Dunham, M. M., et al. 2014, Protostars and Planets VI, 387 
Banzatti, A., Meyer, M. R., Bruderer, S., et al. 2012, ApJ, 745, 90 Baraffe, I., \& Chabrier, G. 2010, A\&A, 521, A44

Benz, A. O., Bruderer, S., van Dishoeck, E. F., et al. 2015, A\&A, 576, A109

Bergin, E. A., Alves, J., Huard, T., \& Lada, C. J. 2002, ApJ, 570, L101

Billot, N., Morales-Calderón, M., Stauffer, J. R., Megeath, S. T., \& Whitney, B. 2012, ApJ, 753, L35

Bisschop, S. E., Fraser, H. J., Öberg, K. I., van Dishoeck, E. F., \& Schlemmer, S. 2006, A\&A, 449, 1297

Black, J. H., \& van Dishoeck, E. F. 1987, ApJ, 322, 412

Blake, G. A., Sandell, G., van Dishoeck, E. F., et al. 1995, ApJ, 441, 689

Boogert, A. C. A., Tielens, A. G. G. M., Ceccarelli, C., et al. 2000, A\&A, 360, 683

Boogert, A. C. A., Hogerheijde, M. R., Ceccarelli, C., et al. 2002, ApJ, 570, 708

Boogert, A. C. A., Pontoppidan, K. M., Knez, C., et al. 2008, ApJ, 678, 985

Butner, H. M., Lada, E. A., \& Loren, R. B. 1995, ApJ, 448, 207

Caselli, P., Myers, P. C., \& Thaddeus, P. 1995, ApJ, 455, L77

Connelley, M. S., \& Greene, T. P. 2010, AJ, 140, 1214

Cook, A. M., Whittet, D. C. B., Shenoy, S. S., et al. 2011, ApJ, 730, 124

Dalgarno, A. 2006, Proc. Nat. Acad. Sci., 103, 12269

Daniel, F., Dubernet, M.-L., Meuwly, M., Cernicharo, J., \& Pagani, L. 2005, MNRAS, 363, 1083

de Graauw, T., Helmich, F. P., Phillips, T. G., et al. 2010, A\&A, 518, L6

Devine, D., Bally, J., Reipurth, B., \& Heathcote, S. 1997, AJ, 114, 2095

Dunham, M. M., Stutz, A. M., Allen, L. E., et al. 2014, Protostars and Planets VI, 195

Emprechtinger, M., Caselli, P., Volgenau, N. H., Stutzki, J., \& Wiedner, M. C. 2009, A\&A, 493, 89

Evans, II, N. J., Lee, J.-E., Rawlings, J. M. C., \& Choi, M. 2005, ApJ, 626, 919

Evans, N. J., Dunham, M. M., Jørgensen, J. K., et al. 2009, ApJS, 181, 321

Fischer, W. J., Megeath, S. T., Stutz, A. M., et al. 2013, Astron. Nachr., 334, 53

Flower, D. R. 1999, MNRAS, 305, 651

Fraser, H. J., Collings, M. P., McCoustra, M. R. S., \& Williams, D. A. 2001, MNRAS, 327, 1165

Gerakines, P. A., Schutte, W. A., Greenberg, J. M., \& van Dishoeck, E. F. 1995, A\&A, 296, 810

Gregersen, E. M., Evans, II, N. J., Zhou, S., \& Choi, M. 1997, ApJ, 484, 256

Gregersen, E. M., Evans, II, N. J., Mardones, D., \& Myers, P. C. 2000, ApJ, 533, 440

Hasegawa, T. I., \& Herbst, E. 1993, MNRAS, 261, 83

Herbig, G. H. 1977, ApJ, 217, 693

Hogerheijde, M. R., \& van der Tak, F. F. S. 2000, A\&A, 362, 697

Hogerheijde, M. R., van Dishoeck, E. F., Salverda, J. M., \& Blake, G. A. 1999, ApJ, 513, 350

Ivezić, Z., Nenkova, M., \& Elitzur, M. 1999, User Manual for DUSTY, Univ. of Kentucky Internal Report, http://www.pa.uky . edu/ moshe/dusty

Johnstone, D., Hendricks, B., Herczeg, G. J., \& Bruderer, S. 2013, ApJ, 765, 133

Jørgensen, J. K. 2004, A\&A, 424, 589

Jørgensen, J. K., Schöier, F. L., \& van Dishoeck, E. F. 2002, A\&A, 389, 908

Jørgensen, J. K., Schöier, F. L., \& van Dishoeck, E. F. 2004, A\&A, 416, 603

Jørgensen, J. K., Visser, R., Sakai, N., et al. 2013, ApJ, 779, L22

Jørgensen, J. K., Visser, R., Williams, J., \& Bergin, E. A. 2015, A\&A, accepted [arXiv: 1504.02974]

Kenyon, S. J., Hartmann, L. W., Strom, K. M., \& Strom, S. E. 1990, AJ, 99, 869

Kim, H. J., Evans, II, N. J., Dunham, M. M., et al. 2011, ApJ, 729, 84

Kim, H. J., Evans, II, N. J., Dunham, M. M., Lee, J.-E., \& Pontoppidan, K. M. 2012, ApJ, 758, 38

Kristensen, L. E., van Dishoeck, E. F., Bergin, E. A., et al. 2012, A\&A, 542, A8

Kryukova, E., Megeath, S. T., Gutermuth, R. A., et al. 2012, AJ, 144, 31
Lada, C. J. 1999, in The Origin of Stars and Planetary Systems, eds. C. J. Lada, \& N. D. Kylafis (Dordrecht: Kluwer), 143

Lee, J.-E. 2007, J. Korean Astron. Soc., 40, 83

Liebhart, A., Güdel, M., Skinner, S. L., \& Green, J. 2014, A\&A, 570, L11

Mardones, D., Myers, P. C., Tafalla, M., et al. 1997, ApJ, 489, 719

Maret, S., Bergin, E. A., \& Lada, C. J. 2006, Nature, 442, 425

McElroy, D., Walsh, C., Markwick, A. J., et al. 2013, A\&A, 550, A36

Minissale, M. 2014, Ph.D. Thesis, Observatoire de Paris-Meudon \& Université de Cergy-Pontoise

Morales-Calderón, M., Stauffer, J. R., Hillenbrand, L. A., et al. 2011, ApJ, 733, 50

Noble, J. A., Congiu, E., Dulieu, F., \& Fraser, H. J. 2012, MNRAS, 421, 768

Öberg, K. I., Fuchs, G. W., Awad, Z., et al. 2007, ApJ, 662, L23

Onishi, T., Mizuno, A., Kawamura, A., Tachihara, K., \& Fukui, Y. 2002, ApJ, 575,950

Ott, S. 2010, in Astronomical Data Analysis Software and Systems XIX, eds. Y. Mizumoto, K.-I. Morita, \& M. Ohishi (San Francisco: ASP), ASP Conf. Ser., 434,139

Owen, J. E., \& Jacquet, E. 2015, MNRAS, 446, 3285

Padoan, P., Haugbølle, T., \& Nordlund, A. 2014, ApJ, 797, 32

Pilbratt, G. L., Riedinger, J. R., Passvogel, T., et al. 2010, A\&A, 518, L1

Pontoppidan, K. M., Boogert, A. C. A., Fraser, H. J., et al. 2008, ApJ, 678, 1005

Poteet, C. A., Pontoppidan, K. M., Megeath, S. T., et al. 2013, ApJ, 766, 117

Qi, C., Öberg, K. I., Wilner, D. J., et al. 2013, Science, 341, 630

Raga, A. C., Velázquez, P. F., Cantó, J., \& Masciadri, E. 2002, A\&A, 395, 647

Rebull, L. M., Cody, A. M., Covey, K. R., et al. 2014, AJ, 148, 92

Roelfsema, P. R., Helmich, F. P., Teyssier, D., et al. 2012, A\&A, 537, A17

Safron, E. J., Fischer, W. J., Megeath, S. T., et al. 2015, ApJ, 800, L5

San José-García, I., Mottram, J. C., van Dishoeck, E. F., et al. 2015, A\&A, submitted

Schmalzl, M., Visser, R., Walsh, C., et al. 2014, A\&A, 572, A81

Schöier, F. L., van der Tak, F. F. S., van Dishoeck, E. F., \& Black, J. H. 2005, A\&A, 432, 369

Scholz, A., Froebrich, D., \& Wood, K. 2013, MNRAS, 430, 2910

Spaans, M., Hogerheijde, M. R., Mundy, L. G., \& van Dishoeck, E. F. 1995, ApJ, 455, L167

Stamatellos, D., Whitworth, A. P., \& Hubber, D. A. 2011, ApJ, 730, 32

Stamatellos, D., Whitworth, A. P., \& Hubber, D. A. 2012, MNRAS, 427, 1182

Stauffer, J., Cody, A. M., Baglin, A., et al. 2014, AJ, 147, 83

Tielens, A. G. G. M., Tokunaga, A. T., Geballe, T. R., \& Baas, F. 1991, ApJ, 381, 181

Tobin, J. J., Hartmann, L., Chiang, H.-F., et al. 2011, ApJ, 740, 45

Tobin, J. J., Hartmann, L., Chiang, H.-F., et al. 2012, Nature, 492, 83

van Dishoeck, E. F., Kristensen, L. E., Benz, A. O., et al. 2011, PASP, 123, 138

van Kempen, T. A., van Dishoeck, E. F., Hogerheijde, M. R., \& Güsten, R. 2009, A\&A, 508, 259

Visser, R., \& Bergin, E. A. 2012, ApJ, 754, L18 (Paper I)

Visser, R., Doty, S. D., \& van Dishoeck, E. F. 2011, A\&A, 534, A132

Vorobyov, E. I., \& Basu, S. 2005, ApJ, 633, L137

Vorobyov, E. I., \& Basu, S. 2010, ApJ, 719, 1896

Vorobyov, E. I., Baraffe, I., Harries, T., \& Chabrier, G. 2013, A\&A, 557, A35

Watson, W. D., \& Salpeter, E. E. 1972, ApJ, 174, 321

Whittet, D. C. B., Cook, A. M., Chiar, J. E., et al. 2009, ApJ, 695, 94

Wilson, T. L. 1999, Rep. Prog. Phys., 62, 143

Yang, B., Stancil, P. C., Balakrishnan, N., \& Forrey, R. C. 2010, ApJ, 718, 1062

Yıldı, U. A., Kristensen, L. E., van Dishoeck, E. F., et al. 2012, A\&A, 542, A86

Yıldı, U. A., Kristensen, L. E., van Dishoeck, E. F., et al. 2013, A\&A, 556, A89

Zasowski, G., Kemper, F., Watson, D. M., et al. 2009, ApJ, 694, 459

Zhu, Z., Hartmann, L., \& Gammie, C. 2009, ApJ, 694, 1045 